\title{
Schulisches Wohlbefinden, Zugehörigkeit und Unterstützung bei Schülerinnen und Schülern im gemeinsamen Unterricht der Sekundarstufe I
}

\author{
Björn Kröske (D)
}

Online publiziert: 24. Januar 2020

(C) Der/die Autor(en) 2020

Zusammenfassung Die vorliegende Studie untersucht, inwiefern im gemeinsamen Unterricht in der Sekundarstufe I (7.-10. Klasse) das schulische Wohlbefinden, die soziale Zugehörigkeit und die empfundene Unterstützung vom sonderpädagogischen Förderbedarf (SPF) in den Bereichen Lernen (SPF-L), emotionale und soziale Entwicklung (SPF-E/S) und geistige Entwicklung (SPF-G) abhängen. Als weitere Einflussgrößen werden der Leistungsdruck und die Förderung zur Zusammenarbeit berücksichtigt. Als Einflussfaktoren für das Wohlbefinden werden darüberhinaus die Zugehörigkeit und die Unterstützung durch die Lehrkräfte betrachtet. Die Ergebnisse der Mehrebenenanalyse zeigen, dass besonders Schüler_innen mit SPF-E/S ein geringeres schulisches Wohlbefinden, weniger soziale Zugehörigkeit und weniger Unterstützung berichten als Schüler_innen ohne SPF. Hingegen ist das psychosoziale Empfinden SPF-L und -G überwiegend vergleichbar mit dem ihrer Mitschüler_innen ohne SPF. Die soziale Unterstützung und Zugehörigkeit tragen zum schulischen Wohlbefinden bei, erklären aber nicht vollständig das geringere Wohlbefinden der Schüler_innen mit SPF-E/S auf, allerdings wirkt sich die Förderung zur Zusammenarbeit in der Klasse positiv auf das psycho-sozialen Erleben aus. Weitergehend wird diskutiert, wie das Erleben von prosozialem Verhalten, speziell bei Schüler_innen mit SPF-E/S, wiederum positiv auf das eigene Sozialverhalten wirkt und folglich ihr Befinden im Unterricht verbessern kann.

Schlüsselwörter Inklusion · sonderpädagogischer Förderbedarf · soziale Zugehörigkeit · Unterstützung · Wohlbefinden · Sekundarstufe

Zusatzmaterial online Zusätzliche Informationen sind in der Online-Version dieses Artikels (https:// doi.org/10.1007/s42010-020-00067-7) enthalten.

B. Kröske $(\bowtie)$

Institut für Erziehungswissenschaften, Pädagogische Psychologie und Gesundheitspsychologie,

Humboldt-Universität zu Berlin, Geschwister-Scholl-Straße 7, 10117 Berlin, Deutschland

E-Mail: kroeskeb@hu-berlin.de 


\title{
School well-being, social affiliation and perceived support for students in inclusive secondary education
}

\begin{abstract}
This study examines the extent to which school well-being, social belonging and perceived support depend on Special Educational Needs (SEN) for learning problems (SEN-L), emotional and social development (SEN-E/S) and mental development (SEN-MD) in joint teaching at lower secondary level (grades 7-10). Further influencing factors are the pressure to perform and teachers' encouragement for cooperation. In addition, social affiliation and support by the teachers are considered as influencing factors for well-being. The results of the multi-level analysis show that especially students with SEN-E/S experience lower school well-being, less social belonging and less support than students without SEN. In contrast, the psycho-social feelings of SEN-L and -MD are largely comparable with those of their classmates without SEN. Social support and social affiliation contribute to school well-being, but do not fully explain the lower well-being of students with SEN-E/S. However, encouraging cooperation in the classroom has a positive effect on psycho-social experience. It is also discussed how the experience of prosocial behaviour, especially in students with SEN-E/S, can have a positive effect on their own social behaviour and consequently improve their well-being in class.
\end{abstract}

Keywords inclusion · special educational needs · social support · well-being · secondary education

\section{Einführung}

Das Ziel von schulischer Inklusion ${ }^{1}$ besteht, neben einer angemessenen Leistungsförderung der Kinder und Jugendlichen, auch darin, die soziale Partizipation aller Schüler_innen zu ermöglichen (Gebhardt und Heimlich 2018; Haeberlin et al. 1989). Die Erlebensqualität sozialer Beziehungen stellt einen grundlegenden Aspekt für das emotionale Wohlbefinden im Unterricht dar (Eder 1995; Hascher 2004). Dieses wird als wesentliches pädagogisches Ziel in der Schule betrachtet (Venetz 2014). Aus diesem Grund wird im vorliegenden Beitrag der Frage nachgegangen, inwieweit zwischen den Schüler_innen mit und ohne sonderpädagogischen Förderbedarf (SPF) Unterschiede im affektiven Wohlbefinden und im sozialen Erleben im gemeinsamen Unterricht in der Sekundarstufe I bestehen.

\footnotetext{
1 Hinsichtlich der Begrifflichkeiten Integration und Inklusion wird in diesem Artikel der Argumentation von Wocken (2009) und Grosche (2015) gefolgt. Sie verweisen auf die komplexe Begriffsverwendung, die uneinheitliche Auslegung und fehlende Trennschärfe der Begriffe Integration und Inklusion. In Berlin und Brandenburg verwenden die jeweiligen Schulbehörden den Begriff Inklusion für die Beschreibung ihrer Unterrichtsformen. Sie charakterisieren dies als gemeinsames, individualisiertes Lernen aller Schüler_innen unabhängig von ihren individuellen Voraussetzungen (Ministerium für Bildung, Jugend und Sport des Landes Brandenburg 2018; Senatsverwaltung für Bildung, Jugend und Familie 2018). In diesem Sinne wird im weiteren Verlauf der Terminus Inklusion verwendet und dieser synonym zur Integration verstanden.
} 


\section{Theorie und empirische Befunde}

Die soziale Zugehörigkeit und die wahrgenommene Unterstützung gelten als wichtige Merkmale zur Beurteilung der pädagogischen Inklusion, zugleich können sie als wesentliche Gründe gesehen werden, warum sich Schüler_innen in einer Klasse wohlfühlen oder nicht (Bossaert et al. 2013; Koster et al. 2009). Durch ihre Unterrichtsführung haben die Lehrkräfte dabei die Möglichkeit Einfluss auf die vorherrschenden Interaktionsstrukturen zu nehmen, was wiederum die gegenseitige Unterstützung der Schüler_innen beeinflussen kann (Patrick et al. 2007). Diese Bemühungen der Lehrkräfte um die gegenseitige Zusammenarbeit der Schüler_innen soll in dieser Untersuchung als Merkmal des Unterrichts zusätzlich berücksichtigt werden. Ein weiterer Aspekt, der als Risiko für Schüler_innen des gemeinsamen Unterrichts diskutiert wird, stellt die leistungsbezogene Überforderung dar (Klicpera 2007). Hoher Leistungsdruck, insbesondere bei Schüler_innen mit schwächeren Leistungen und abweichendem Verhalten, steht in Verbindung mit einer erhöhten gesundheitlichen Belastung und beeinträchtigt das emotionale Erleben in der Klasse (Schubarth und Speck 2008). Daher soll auch der Leistungsdruck als Prädiktor in diese Untersuchung einbezogen werden.

\subsection{Wohlbefinden von Schüler_innen mit SPF}

Das schulische Wohlbefinden umfasst neben den Dimensionen positive Einstellung zur Schule und Freude in der Schule nach Hascher (2004) auch leistungsbezogene Konstrukte (z.B. schulisches Selbstkonzept) und Stresskomponenten (schulbezogene Sorge und körperliche Beschwerden). Venetz (2014) beschreibt das subjektive Wohlbefinden als Ausmaß der selbstwahrgenommenen, aktuellen Zufriedenheit und hebt die Anwesenheit positiver und die Abwesenheit negativer emotionaler Erlebenszustände hervor (Venetz et al. 2010). Vergleichbar damit und in Anlehnung an Hascher und Lobsang (2004) unterscheiden Schwinger et al. (2015) zwei Komponenten des schulischen Wohlbefindens. Das körperliche schulische Wohlbefinden wird durch psychosomatische Indikatoren (z. B. Bauchschmerzen) bestimmt, während das affektive schulische Wohlbefinden durch positive bzw. negative Gefühle (z. B. Freude, Zufriedenheit, Leistungsangst) gekennzeichnet ist. In der vorliegenden Untersuchung soll der Schwerpunkt auf der letztgenannten Komponente liegen.

Insgesamt sind Untersuchungen zum Thema schulisches Wohlbefinden im inklusiven Schulunterricht rar. Entsprechende Studien konzentrieren sich vornehmlich auf den Grundschulbereich (Stelling 2017; Wild et al. 2015), untersuchen die Fragestellung ausschließlich qualitativ (Külker et al. 2017) oder liegen im deutschen Sprachraum überwiegend für Österreich oder die Schweiz vor (Haeberlin et al. 1991; Schwab 2014a, 2015b; Venetz 2014). Diese Arbeiten kommen mehrheitlich zu dem Fazit, dass es in inklusiven Schulsettings im Wohlbefinden bzw. psychosozialen Erleben von Schüler_innen mit SPF keine bedeutsamen Unterschiede im Vergleich zu Schüler_innen ohne SPF gibt. Lediglich Haeberlin et al. (1991) fanden bei Schüler_innen mit Lernbehinderung ein geringeres subjektives Wohlbefinden im Vergleich zu Schüler_innen ohne Lernbehinderung. Hingegen weisen internationale Untersuchungen außerhalb des deutschen Sprachraums überwiegend ein schlechte- 
res emotionales Wohlbefinden bei Schüler_innen mit SPF nach (Barnes und Harrison 2017; Bryan et al. 2004; Gaspar et al. 2016; Lackaye et al. 2006; McCoy und Banks 2012).

Eine der wenigen Untersuchungen in Deutschland in der Sekundarstufe stammt von Sauer et al. (2007). Diese fanden in der 6.-8. Klassenstufe bei der emotionalen Integration (z.B. „Ich gehe gerne in die Schule“) keine Unterschiede zwischen Schüler_innen mit SPF im Bereich Lernen und ohne SPF. Untersuchungen, die mehrere Formen des Förderbedarfs differenziert betrachten, stammen z. B. von Venetz et al. (2010). Sie verglichen Schweizer Schüler_innen mit Schulleistungsschwäche und solche mit Verhaltensauffälligkeit und fanden keine Gruppenunterschiede im affektiven Wohlbefinden. McCoy und Banks (2012) befragten 8578 9-jährige irische Schüler_innen und kommen zu dem Ergebnis, dass solche mit Förderbedarf im Bereich Lernen und Verhalten eine höhere Wahrscheinlichkeit aufweisen, die Schule nicht zu mögen, als solche mit anderen Förderschwerpunkten. Die Autoren weisen darauf hin, dass besonders Jungen und Schüler_innen aus Familien mit niedrigerem sozioökonomischem Status über ein geringeres Wohlbefinden berichten und daher dieses Merkmal in vergleichbaren Untersuchungen kontrolliert werden sollte. Grüning (2011) untersuchte mittels Beobachtungsverfahren das emotionale Wohlbefinden von deutschen Grundschüler_innen mit intellektuell-kognitiven Beeinträchtigungen. Er kommt zur Einschätzung, dass für diese Gruppe das aktive und freudvolle Lernen im integrativen Unterricht trotz gefundener Differenzen, wie z. B. bei Unsicherheit in den Pausen, grundlegend gegeben und als gut zu bewerten ist.

Der Forschungsüberblick zeigt, dass bisher wenig bekannt ist zum Wohlbefinden von Deutschen Schüler_innen mit SPF im gemeinsamen Unterricht in Klassen der Sekundarstufe. Basierend auf den deutschsprachigen Befunden, die überwiegend aus der Primarstufe stammen (Sauer et al. 2007; Schwab et al. 2015b; Venetz 2014; Venetz et al. 2010; Wild et al. 2015), soll geprüft werden, ob auch in der Sekundarstufe I keine Unterschiede im affektiven Wohlbefinden in Abhängigkeit vom SPF $\mathrm{zu}$ finden sind.

\subsection{Soziale Zugehörigkeit in Inklusionsklassen}

In der Forschung haben sich für die soziale Partizipation aller Schüler_innen unterschiedliche Begrifflichkeiten etabliert. Koster et al. (2009) analysierten 62 Artikel zu den Konzepten soziale Integration, soziale Inklusion und soziale Teilhabe und schlussfolgerten, dass diese Begriffe synonym verwendet werden. Zur Operationalisierung der sozialen Dimension von Inklusion erarbeiteten Koster et al. (2009) vier wesentliche Facetten. Diese beinhalten (1) positive Interaktionen zwischen den Schüler_innen mit und ohne SPF (z.B. zusammen spielen oder gemeinsam arbeiten), (2) Freundschaften zwischen Schüler_innen mit und ohne SPF, (3) die eigene Wahrnehmung der sozialen Partizipation in der Klasse (z. B. das Gefühl von Einsamkeit, das soziale Selbstkonzept) und (4) die Akzeptanz durch die Mitschüler_innen (z.B. soziale Unterstützung oder Ablehnung, Bullying, Zurückweisungen). In den meisten Arbeiten, welche sich mit der sozialen Teilhabe oder Integration beschäftigen, werden besonders die Facetten eins, zwei und vier mithilfe von soziometrischen und netzwerkanalytischen Methoden über den gegenseitigen Wahlstatus erfasst. Hier 
zeigt sich übergreifend, dass Schüler_innen mit SPF im Vergleich zu Schüler_innen ohne SPF häufig ein höheres Ausgrenzungsrisiko haben (Bless und Mohr 2007; Henke et al. 2017; Huber 2008; Krull et al. 2014a; Kulawiak und Wilbert 2015; Pijl und Frostad 2010; Schwab 2019). Betrachtet man speziell Schüler_innen mit intellektuell-kognitiven Einschränkungen, zeigt sich ebenso, dass diese weniger gut in ihren Klassen eingebunden sind (Bakker et al. 2007; Dumke und Schäfer 1993; Garrote 2017; Kemp und Carter 2002; Siperstein et al. 2009). Vergleichbares lässt sich auch zu Schüler_innen mit Lernproblemen finden (Estell et al. 2008; Frederickson und Furnham 2004; Lorger et al. 2015). Besonders Schüler_innen mit sozial-emotionalen Problemen werden auffällig oft ausgegrenzt bzw. sind schlecht eingebunden (Bierman et al. 1993; Chang 2004; Mand 2007; Nepi et al. 2013).

Vergleichbar mit der dritten Facette von Koster et al. (2009) definieren Doubt und McColl (2003) soziale Integration als ein Gefühl der Zugehörigkeit, welches durch Akzeptanz und gleichberechtigte Teilhabe erzeugt wird. Dieses Gefühl der Zugehörigkeit ist nach Haeberlin et al. (1999) von der eigenen Wahrnehmung und Beurteilung der eigenen Beziehungen zu den Mitschüler_innen abhängig (Schwab 2014b). Krawinkel et al. (2017) fanden, dass Grundschüler_innen mit SPF auch in der eigenen Wahrnehmung ihre soziale Partizipation im Vergleich zu Kindern ohne SPF schlechter einschätzten. Weitere Studien, die die selbsteingeschätzte soziale Partizipation erhoben, jedoch alle im Primarbereich (1.-6. Klassenstufe), kommen zu vergleichbaren Ergebnissen (Cambra und Silvestre 2003; Huber und Wilbert 2012; Schwab et al. 2015a). Es liegen allerdings auch Untersuchungen vor, in denen kein bedeutsamer Unterschied belegt werden konnte (Koster et al. 2010; Martschinke et al. 2012; Wild et al. 2015). Krawinkel et al. (2017) weisen darauf hin, dass einige der Studien in diesem Bereich teilweise mit methodischen Einschränkungen verbunden sind, z. B. aufgrund recht kleiner Stichproben (Martschinke et al. 2012; Schwab et al. 2015a) oder der Operationalisierung des Förderbedarfs (Huber und Wilbert 2012).

Bisher gibt es nur vereinzelt Studien, in denen die soziale Eingebundenheit zwischen verschiedenen Formen von Förderbedarfen verglichen wurde. Wocken (1993) fand unter 1055 Hamburger Schüler_innen (von Primar bis Sekundarstufe) heraus, dass Schüler_innen mit Verhaltensproblemen die am meisten abgelehnte und isolierte Gruppe im Vergleich zu denen mit kognitiven Einschränkungen, körperlichen Einschränkungen oder Lernproblemen sind. Weitere Untersuchungen aus dem Primarbereich berichten ebenso davon, dass die Schüler_innen mit Verhaltensschwierigkeiten am stärksten ausgegrenzt sind (Avramidis 2010; Bossaert et al. 2015; Krull et al. 2014b; Nepi et al. 2013). Allerdings fanden Bossaert et al. (2015) in ihrer Untersuchung, in die auch Daten aus Belgien und den Niederlanden einflossen, in der Kohorte der norwegischen Schüler_innen keinen Unterschied in den Akzeptanzwerten der Schüler_innen mit SPF, was auch SPF-E/S einschließt. Dies war auch bei Koster et al. (2010) der Fall. Ein Vergleich zwischen Schüler_innen mit verschiedenen Kategorien von Förderbedarfen (Verhalten, Autismus, Motorik, kognitiv, Sprache) ergab keine signifikanten Unterschiede in der sozialen Partizipation.

Diese geringe Anzahl an Studien zur sozialen Situation von Schüler_innen im gemeinsamen Unterricht, die auch zwischen verschiedenen Formen von Förderbedarfen unterscheiden, zeigt nicht nur einen weiteren Forschungsbedarf in diesem 
Bereich auf, sondern auch, dass die Situation von Schüler_innen mit SPF in der Sekundarstufe in deutschen Schulen bisher kaum Berücksichtigung gefunden hat. Daher soll geprüft werden, ob in der Sekundarstufe I die erlebte Zughörigkeit von der Art des SPF abhängig ist.

\subsection{Unterstützung in Inklusionsklassen}

Die wahrgenommene soziale Unterstützung ist ein wesentliches Element der individuellen Belastungsbewältigung (Waligora 2002) und hat eine protektive Wirkung vor den negativen Folgen von Stress und einen positiven Einfluss auf das Wohlbefinden (Langford et al. 1997). Dumont und Provost (1999) definieren soziale Unterstützung als ein Konzept, das die erhaltene Unterstützung (informativ, emotional und instrumental) und die Quellen der Unterstützung (Freunde, Familie, Fremde) beinhaltet. Anders als die soziale Partizipation, welche die soziale Netzwerkstruktur umfasst, und die (emotionale) Stärke der Verbindung (u.a. wie oft und lange man Kontakt hat), geht es bei der sozialen Unterstützung um die Funktion der Kontakte (z.B. Informationen, Hilfe, Beistand erhalten). Ein vorhandenes soziales Netzwerk mit hoher emotionaler Qualität in einer Atmosphäre der Unterstützung (positives soziales Klima) wird als Voraussetzung für unterstützendes Verhalten gesehen (Langford et al. 1997).

Es gibt nur wenige Untersuchungen zur sozialen Unterstützung im inklusiven Unterricht. Wenz-Gross und Siperstein (1998) zeigten in der 6.-8. Klassenstufe $(N=437)$, dass Schüler_innen mit Lernproblemen (bis hin zur Lernbehinderung) mehr Stress und weniger Unterstützung durch Gleichaltrige erleben als diejenigen ohne Lernschwierigkeiten. Bei Martínez (2006) konnte der Faktor Lernbehinderung in derselben Altersstufe $21 \%$ der Varianz der sozialen Unterstützung erklären. Humphrey und Symes (2010) befragten 120 englische Schüler_innen der Secondary School, von denen jeweils ein Drittel eine Diagnose für Dyslexie oder Autismus aufwiesen. Hinsichtlich der wahrgenommenen Unterstützung zeigte sich, dass die Schüler_innen mit Autismus, nicht aber die mit Dyslexie, von weniger Unterstützung durch ihre Klassenkameraden berichteten. Eine Untersuchung in zwei italienischen Grundschulen von Nota et al. (2005) zeigte, dass die Bereitschaft, Mitschüler_innen mit intellektuell-kognitiven Einschränkungen zu helfen, deutlich höher ist, als mit ihnen befreundet sein zu wollen. Die vorliegende Untersuchung soll einen Beitrag zur weiteren Klärung der Rolle der wahrgenommenen Unterstützung für das affektive schulische Wohlbefinden und die soziale Zugehörigkeit leisten.

Auch die Lehrkräfte haben die Möglichkeit gegenseitige Unterstützung der Schüler_innen zu fördern. Für das Unterrichten in heterogenen Lerngruppen wird empfohlen, dass die Anforderungen und Aufgaben sinnvoll differenziert werden, dabei aber die Schüler_innen auch in heterogenen Gruppen lernen und kooperierend zusammenzuarbeiten (Green und Green 2005; Vehkakoski 2012; Werning und Löser 2010). Die Lehrkräfte sollen also auch die Zusammenarbeit durch ihre Gestaltung des Unterrichts fördern. Die Forschung im Bereich der multikulturellen Inklusion zeigt, dass eine Förderung von positiver Einstellung zu Pluralismus und Wertschätzung gegenüber Diversität die wahrgenommene Diskriminierung und Viktimisierung reduzieren kann (Bayram Özdemir und Stattin 2014; Morin et al. 2013). Eine geziel- 
te Förderung von positiven Einstellungen zur Heterogenität wurde auch mit einem höheren Wohlbefinden in Verbindung gebracht und kann die wahrgenommene Distanz in der Klasse reduzieren (Schachner et al. 2015, 2016). Um zu prüfen, ob diese Ergebnisse auch auf die sonderpädagogische Inklusion übertragbar sind, soll analysiert werden, ob die wahrgenommene Förderung zur gegenseitigen Zusammenarbeit durch die Lehrkräfte und die wahrgenommene Unterstützung der Schüler_innen das schulische Wohlbefinden der Schüler_innen mit SPF positiv beeinflussen.

\subsection{Leistungsdruck}

Die Unterstützungsbereitschaft kann von den Lehrkräften nicht nur gefördert werden, indem diese zum Zusammenarbeiten direkt auffordern oder mit entsprechenden Übungen kooperatives Lernen fördern, sondern auch der wahrgenommene Leistungsdruck hängt mit der empfundenen Unterstützung zusammen (Lätsch 2017; Torsheim et al. 2003). Hascher (2004) versteht unter Leistungsdruck ein Merkmal des Unterrichts, welches durch die Lehrkraft geprägt ist. Dies äußert sich z. B. im schnellen Fortschreiten im Unterrichtsstoff und einer Fülle von Arbeitsaufträgen, so dass individuelle Lernbedürfnisse unberücksichtigt bleiben.

In einem Überblick über sechs empirische Studien mit Stichproben, mehrheitlich aus der Schweiz (aber auch aus Deutschland, Niederlande und Tschechien), identifiziert Hascher (2004) den Leistungsdruck in den Klassenstufen 7-9 als einen der relevantesten Prädiktoren für das Wohlbefinden in der Schule. In einer Längsschnittstudie (6.-7. Klassenstufe) von Hagenauer (2011) mit Schüler_inn aus Salzburg und Umgebung wurde zwar ein Zusammenhang von Leistungsdruck mit geringer Lernfreunde (affektives Wohlbefinden) gefunden, jedoch konnte der Leistungsdruck in der 6. Klasse die Lernfreunde in der 7. Klasse nicht zuverlässig vorhersagen. Bei deutschen Schüler_innen (Klassenstufe 7) wurde von Gruehn (1995) lediglich ein geringer Zusammenhang zwischen Leistungsdruck und Schulfreude nachgewiesen. Eine weitere Untersuchung an 7625 österreichischen Schüler_innen von Eder (2007) in der 4.-12. Klasse zeigte, dass u.a. Leistungsdruck ein bedeutsamer Prädiktor für das Wohlbefinden in allen Klassenstufen ist. Studien, welche sich mit diesem Zusammenhang im gemeinsamen Unterricht beschäftigen, konnten nicht gefunden werden.

Jedoch gibt es Hinweise, dass in leistungsheterogenen Gruppen der Leistungsdruck die Zusammenarbeit beeinflussen kann. Hoher Leistungsdruck sorgt dafür, dass in einer Lerngruppe Personen eher ausgeschlossen werden, wenn sie im Konflikt mit dem effektiven Funktionieren der Gruppe (z. B. bei Gruppenarbeiten) stehen. Bei hohem Leistungsdruck wäre dies für leistungsschwächere Schüler_innen der Fall, da sie das Erzielen hoher Leistungen für die Gruppe erschweren würden. Stehen die außenstehenden Personen hingegen nicht mit dem effektiven Funktionieren der Gruppe in Konflikt, dann erfolgt deren Aufnahme bereitwilliger (Killen und Stangor 2001; Richardson et al. 2012). Somit scheint die Integration und Unterstützung von Leistungsschwächeren stark vom Leistungsanspruch in der Klasse abzuhängen. Personen mit aggressivem und hyperaktivem Verhalten werden hingegen auch bei geringerem Leistungsdruck stärker abgelehnt (Gasser et al. 2017; Richardson et al. 2014). Hierzu lässt sich vermuten, dass dies darauf zurückzuführen sein könnte, 
dass aggressives und hyperaktives Verhalten, unabhängig vom Leistungsanspruch, die Zusammenarbeit erschwert.

Im Folgenden soll bezüglich des Leistungsdrucks geprüft werden, ob auch dieser in Abhängigkeit vom Förderstatus variiert, inwiefern ein Einfluss auf das Wohlbefinden bestätigt werden kann und ob der Leistungsdruck eine erklärende Größe für die empfundene Unterstützung und die Zugehörigkeit ist.

\section{Forschungsfragen und Hypothesen}

In der vorliegenden Studie soll der Frage nach der sozio-emotionalen Situation von Schüler_innen der 7.-10. Klassenstufe im gemeinsamen Unterricht nachgegangen werden. Das Forschungsdesiderat in diesem Bereich besteht vor allem im Mangel an Untersuchungen in deutschen Schulkassen der Sekundarstufe und in der differenzierten Betrachtung der verschiedenen Formen des SPF. In den Bundesländern Berlin und Brandenburg wird zwischen acht sonderpädagogischen Förderschwerpunkten unterschieden (für einen Überblick siehe: Senatsverwaltung für Bildung Jugend und Familie 2017; bzw. Ministerium für Bildung Jugend und Sport 2018). In der folgenden Untersuchung werden drei Förderschwerpunkte differenziert ausgewertet: Förderschwerpunkt Lernen (SPF-L), geistige Entwicklung (SPF-G) sowie emotionale und soziale Entwicklung (SPF-E/S). Diese gehören zu den am stärksten vertretenen Förderschwerpunkten, die zusammengefasst etwa $70 \%$ aller Schüler_innen mit SPF in Deutschland ausmachen (Sekretariat der Ständigen Konferenz der Kultusminister der Länder in der Bundesrepublik Deutschland 2018).

Für die nachfolgende Untersuchung werden das Wohlbefinden (operationalisiert durch vier abhängige Variablen: Wohlbefinden im Unterricht und in der Pause sowie positives und negatives affektives schulisches Wohlbefinden), die soziale Zugehörigkeit und die empfundene Unterstützung als Zielgrößen betrachtet. Nach Kontrolle des Geschlechts, des Alters und des subjektiven sozioökonomischen Status soll der Einfluss auf diese 6 Zielvariablen geprüft werden.

Modell 1 Der Einfluss des Status des Sonderpädagogischen Förderbedarfs auf die Zielvariablen soll geprüft werden.

Modell 2 Das Modell soll um die Einflüsse der Größen des Sozialklimas (wahrgenommene Zugehörigkeit, empfundene Unterstützung) erweitert und geprüft werden.

Modell 3 Das Modell soll um die Einflüsse der Merkmale der Lernumgebung (Leistungsdruck, Förderung zur Zusammenarbeit durch die Lehrkräfte) erweitert und geprüft werden. 
Tab. 1 Details zur Stichprobe

\begin{tabular}{|c|c|c|c|c|c|c|c|c|c|}
\hline & \multirow[b]{2}{*}{$N$} & \multicolumn{2}{|c|}{$\begin{array}{l}\text { Geschlecht } \\
\text { (Anteil in \%) }\end{array}$} & \multirow{2}{*}{$\begin{array}{l}\text { Mittl. } \\
\text { Alter } \\
(\mathrm{SD})\end{array}$} & \multirow{2}{*}{$\begin{array}{l}\text { Anteil } \\
\text { Migrations- } \\
\text { hintergrund } \\
\text { (Anteil in \%) }\end{array}$} & \multicolumn{4}{|c|}{ Anzahl SPF } \\
\hline & & Männlich & Weiblich & & & SPF-L & $\begin{array}{l}\text { SPF- } \\
\text { E/S }\end{array}$ & SPF-G & $\begin{array}{l}\text { Restl. } \\
\text { SPF }\end{array}$ \\
\hline 7. Klasse & 389 & 49,9 & 49,6 & $\begin{array}{l}12,35 \\
(0,64)\end{array}$ & 28,5 & 11 & 21 & 13 & 4 \\
\hline 8. Klasse & 369 & 52,6 & 46,9 & $\begin{array}{l}13,43 \\
(0,70)\end{array}$ & 19,0 & 18 & 19 & 3 & 12 \\
\hline 9. Klasse & 421 & 52,5 & 45,8 & $\begin{array}{l}14,42 \\
(0,74)\end{array}$ & 29,0 & 18 & 16 & 6 & 13 \\
\hline 10. Klasse & 320 & 53,1 & 45,9 & $\begin{array}{l}15,5 \\
(0,78)\end{array}$ & 26,6 & 5 & 8 & 6 & 7 \\
\hline Gesamt & 1499 & 52,0 & 47,1 & $\begin{array}{l}13,87 \\
(1,34)\end{array}$ & 25,9 & 52 & 64 & 28 & 36 \\
\hline
\end{tabular}

\section{Methode}

\subsection{Stichprobe}

Die Untersuchung erfolgte in 85 Klassen der Klassenstufen 7-10 an acht Schulen in Berlin und Brandenburg. Bei allen Schulen handelt es sich um Schultypen der Sekundarbildung I (ohne Gymnasium), die Schüler_innen mit und ohne SPF gemeinsam unterrichten. Die Teilnahme der Schulen und der Schüler_innen erfolgte freiwillig. Von 1540 erhaltenen Fragebögen konnten 1499 ausgewertet werden. Details zur Stichprobe sind in Tab. 1 abgebildet. Die Angaben zum diagnostizierten SPF erfolgten durch das Sekretariat der Schulleitung oder durch kundiges Lehrpersonal. In drei Fällen wurden zwei Förderschwerpunkte angegeben, wobei es sich jeweils um SPF-Autismus mit SPF-L handelte. Da in diesen Fällen keine eindeutige Zuordnung zu einem der drei in dieser Untersuchung berücksichtigten Förderschwerpunkte möglich war, wurde darauf verzichtet, und die Daten wurden der Sammelkategorie restliche SPF zugeordnet.

\subsection{Durchführung}

Die Befragung fand im 4. Quartal 2017 statt. In den Schulen wurden alle Klassen von der 7. bis zur 10. befragt. Die Erhebung in den Klassen erfolgte durch geschulte Testleiter_innen im Beisein von Lehrkräften im Unterricht mittels Fragebogen. Besonders bei Schüler_innen mit SPF-G wurde Unterstützung geboten, wobei diese (auf eigenen Wunsch der jeweiligen Schüler_innen) meist durch schulisches Betreuungspersonal erfolgte, welches die Fragen vorlas. Die Einführung und Anweisungen zum Ablauf der Befragung wurden durch die Testleiter_innen vorgelesen, und diese standen die ganze Befragung über zur weiteren Unterstützung zur Verfügung. Für die Erhebung wurde eine Unterrichtsstunde eingeplant. Die Schüler_innen benötigten für das Ausfüllen der Fragebögen in der Regel zirka 20 min. 


\subsection{Statistische Analysen}

\subsubsection{Umgang mit fehlenden Werten}

Die Anzahl an fehlenden Werten bei den Skalenitems liegt im Mittel bei 3,5\% $(\max .5,8 \%)$. Der MCAR-Test nach Little $\left(\mathrm{X}^{2}(183)=222,51 ; p=0,03\right)$ zeigt an, dass die fehlenden Werte nicht vollständig zufällig sind. Da jedoch nach grafischer Betrachtung und inhaltlicher Reflexion kein offensichtliches Muster in den fehlenden Werten (im Sinne von Not missing at random; NMAR) erkannt werden kann, werden die fehlenden Skalenwerte (mit Ausnahme von nominalskalierten Variablen wie z. B. Geschlecht) EM-imputiert.

\subsubsection{Hinweise zu den Analysen}

Für die deskriptiven Statistiken und Korrelationsanalysen wird SPSS 25 (IBM Corp. 2017) genutzt. Die Analyse der Messinstrumente erfolgt darüber hinaus mittels AMOS 25 (Arbuckle 2017). Für die Mehrebenenanalysen wird R (R Development Core Team 2008), unter Verwendung der Pakte lme4 (Bates et al. 2015) und lmerTest (Kuznetsova et al. 2017), genutzt.

Es werden sechs Modelle mit verschiedenen abhängigen Variablen (positives und negatives affektives schulisches Wohlbefinden, Wohlbefinden im Unterricht und in der Pause sowie die soziale Zugehörigkeit und empfundene Unterstützung) geschätzt und diese Modelle jeweils schrittweise um weitere Variablen erweitert. Neben den Kontrollvariablen Geschlecht, Alter, subjektiver sozioökonomischer Status und Sprache werden auf der Individualebene der SPF-Status, die soziale Zugehörigkeit, die empfundene Unterstützung, der Leistungsdruck und die wahrgenommene Förderung zur Zusammenarbeit durch die Lehrkräfte berücksichtigt. Die letzten beiden Variablen werden zusätzlich auch auf der Klassenebene aufgenommen, da es sich um Unterrichtsmerkmale handelt, welche die Lernumwelt aller Mitglieder einer Gruppe (also der Klasse) betreffen. Die Interklassenkorrelationen der abhängigen Variablen zeigen niedrige Werte von $\rho=0,02-0,06$. Diese machen aber eine Mehrebenenanalyse nicht obsolet (Cohen et al. 2003). Durch die geringe Varianz auf Klassenebene ist allerdings die Beobachtung von relevanten Effekten weniger wahrscheinlich.

Zur besseren Vergleichbarkeit der Ergebnisse werden die Variablen zuvor transformiert. Bei den nominalskalierten Variablen erfolgt eine Dummykodierung (in 0 oder 1) (SPF-E/S; SPF-L; SPF-G: 1 = Förderbedarf jeweils vorhanden; Geschlecht: 1=männlich; Sprache: 1=Deutsch). Beim SPF gilt die Gruppe ohne Förderbedarf als Referenzkategorie. Die weiteren Variablen werden am Gesamtmittelwert zentriert, um die Schätzwerte besser interpretierbar zu machen. Für die Prädiktoren Leistungsdruck und wahrgenommene Förderung zur Zusammenarbeit durch die Lehrkräfte, welche auf der Schülerebene als individualer Faktor und auf Klassenebene als Gruppenfaktor berücksichtig werden, werden die Werte nach der Empfehlung von Enders und Tofighi (2007) auf der Schülerebene am Gruppenmittel und auf der Klassenebene am Gesamtmittelwert zentriert.

Effektstärken von Korrelationen werden nach den Empfehlungen von Cohen (1988) interpretiert. Da es für Mehrebenenanalyse kein direktes Äquivalent 
zum Bestimmtheitsmaß $\mathrm{R}^{2}$ aus der linearen Regression gibt, wurden verschiedene pseudo- $\mathrm{R}^{2}-\mathrm{Maße}$ vorgeschlagen. Diese können analog $\mathrm{zu} \mathrm{R}^{2}$ interpretiert werden und dienen der Bestimmung der Varianzaufklären. Nakagawa und Schielzeth (2013) entwickelten hierzu zwei solche pseudo- $R^{2}$. Mit dem marginalen $R^{2}\left(R^{2}{ }_{G L M M(m)}\right)$ wird die Varianz errechnet, die durch alle festen Effekte (also die aufgenommen Prädiktoren) im Mehrebenenmodell erklärt wird. Das konditionale $\mathrm{R}^{2}\left(\mathrm{R}^{2}{ }_{\mathrm{GLMM}(\mathrm{c})}\right)$ gibt die erklärte Varianz für das Gesamtmodell an. Hier wird dabei zusätzlich die Streuung im Intercept zwischen den Ebenen (hier also den Klassen) berücksichtigt.

Der Datensatz für die Mehrebenenanalyse wird auf die Personen mit SPF-L, SPF-G, SPF-E/S und Personen ohne SPF beschränkt. Dieser Datensatz umfasst $N=1444$ Schüler_innen (kein SPF: 1302; SPF-L: 51, SPF-G: 28, SPF-E/S: 63).

\subsection{Instrumente}

In diesem Abschnitt werden die Messinstrumente kurz beschrieben und deren wesentliche psychometrischen Kennwerte berichtet. Darüber hinaus wurde die Struktur der Skalen (mittels CFA) und die Invarianz der Messinstrumente überprüft. Letzteres ergab keine bedeutsamen Unterschiede im Messverhalten zwischen den SPFGruppen. Im Online-Anhang sind weitere Informationen zu den Messinstrumenten und deren Items hinterlegt. Dort finden Sie auch die Ergebnisse der CFA.

\subsubsection{Affektives schulisches Wohlbefinden}

In Anlehnung an Venetz et al. (2010) werden zwei Komponenten des affektiven schulischen Wohlbefindens differenziert: die Anwesenheit positiver und die Abwesenheit negativer emotionaler Erlebenszustände. Es werden hierzu Skalen von Schwinger et al. (2015) genutzt, die dieses Instrument bei Drittklässlern mit SPF-L erprobt haben. Ursprünglich wurde mit drei Items das positive und mit vier Items das negative schulische Wohlbefinden erfasst. Auf einer 4-stufigen Skala (,,(fast) nie“, ,selten“, „oft", ,(fast) immer“) sollten die Schüler_innen angeben, wie oft sie im letzten Jahr in der Schule z. B. fröhlich oder ängstlich waren. Aufgrund der Ergebnisse der konfirmatorischen Faktorenanalyse (CFA; siehe Anhang) wird das 4. Item (gelangweilt) der negativen Skala entfernt, und es ergibt sich eine akzeptable Passung. Die interne Konsistenz für das positive Wohlbefinden liegt bei $\alpha=0,70$. Die Interklassenkorrelationen (ICC), die den Anteil der Gesamtvarianz angibt, der auf Unterschiede zwischen den Klassen entfällt, liegt bei $\rho=0,03$. Für das negative Wohlbefinden wurde ein Wert von $\alpha=0,50$ und ein ICC von $\rho=0,04$ ermittelt. Neben dem affektiven Wohlbefinden wurden die Schüler_innen auch direkt danach gefragt, wie oft sie sich im letzten Jahr im Unterricht bzw. in der Pause wohlfühlten (,,(fast) nie“, ,,selten“, ,oft", ,(fast) immer“).

\subsubsection{Soziale Zugehörigkeit}

Die Erfassung der sozialen Zugehörigkeit orientierte sich an den Items von Wild et al. (2015). Es wurde u.a. danach gefragt, mit wie vielen (,keinem“, ,wenigen“, „den meisten“, ,(fast) allen“) Schüler_innen die Befragten gerne zusammen oder 
befreundet sind. Bei der CFA zeigen sich gute Fit-Werte, die interne Konsistenz liegt bei $\alpha=0,78$ und die Interklassenkorrelation bei $\rho=0,07$.

\subsubsection{Empfundene Unterstützung}

In Anlehnung an die Student Personal Perception of Classroom Climate (SPPCC; Rowe et al. 2010) und die Landauer Skalen zum Sozialklima (LASSO (4-13); Saldern und Littig 1987) wurden vier Items verfasst, die jeweils die empfundene Unterstützung erfassen sollen (z. B. „Wenn ich jemanden in meiner Klasse um Hilfe bitte, wird mir gerne geholfen."). Zur Beurteilung wurde eine 4-stufige Likert-Skala gewählt (,trifft nicht zu“ bis ,trifft genau zu“). Die Skala erreicht eine zufriedenstellende interne Konsistenz von $\alpha=0,76$ und ein ICC von $\rho=0,07$.

\subsubsection{Förderung zur Zusammenarbeit durch die Lehrkräfte}

Die wahrgenommene Förderung zur Zusammenarbeit durch die Lehrkräfte soll aus Sicht der Schüler_innen erfassen, wie viele ihrer Lehrkräfte sie dazu ermutigen (,,keine“, ,,wenige“, „,die meisten“, ,,alle“), dass sich die Schüler_innen untereinander unterstützen und verstehen. Die Items sind angelehnt an die Subskala Teachers' support for interdependence von Schachner et al. (2016). Sechs von acht Items, die sich auf interkulturelle Interdependenz beziehen, wurden so umformuliert, dass sie auf die Zusammenarbeit im Allgemeinen Bezug nehmen (z.B. „Unsere Lehrer möchten, dass sich alle untereinander helfen, wenn einer etwas nicht versteht."). Inhaltliche Kriterien wie auch statistische Kennwerte sprechen dafür, Item 5 und 6 als eigenen Faktor zu betrachten, wobei dieses 2-faktorielle Modell sehr gute FitMaße erzielt. Im weiteren Verlauf wird nur der erste Faktor genutzt, der sich aus den ersten vier Items zusammensetzt. Hier wird eine interne Konsistenz von $\alpha=0,77$ und Interklassenkorrelation von $\rho=0,16$ erreicht.

\subsubsection{Leistungsdruck}

Zur Erfassung des wahrgenommenen Leistungsdrucks wurde auf die Subskala aus den Landauer Skalen zum Sozialklima (LASSO 4-13; Saldern und Littig 1987) zurückgegriffen und sprachlich angepasst (z.B. „Wenn ich nicht am Wochenende lerne, komme ich im Unterricht kaum noch mit.“). Von den ursprünglich sieben Items wurden aus inhaltlichen Gründen zwei entfernt. Cronbachs Alpha erreicht einen Wert von $\alpha=0,76$, der ICC liegt bei $\rho=0,04$.

\subsubsection{Subjektiver sozioökonomischer Status und demografische Variablen}

Der subjektive sozioökonomische Status wird in die Analysen als Kontrollvariable mit einbezogen, da dieser mit dem schulischen Wohlbefinden (Surachman et al. 2018), den Peer-Beziehungen (Oswald und Krappmann 2004) und mit dem SPF zusammenhängt (Kocaj et al. 2014). Für eine ökonomische Erhebung des sozioökonomischen Status aus subjektiver Sicht wurde auf die MacArthur-Scale zurückgegriffen (Adler und Stewart 2007; Hoebel et al. 2015). Hierbei soll die eigene Familie 
auf einer 10-stufigen Leiter eingeordnet werden, bei der ganz oben die Familien in Deutschland mit dem meisten Geld, der höchsten Bildung und den besten Jobs stehen und unten die mit dem wenigsten Geld, Bildung etc. Als weitere Kontrollvariablen wurden die manifesten Größen Geschlecht, Alter (in Jahren) und die Sprache, die zu Hause am meisten gesprochen wird (als Indikator für einen Migrationshintergrund), erfasst.

\section{Ergebnisse}

\subsection{Mittelwerte und Korrelationen}

In Tab. 2 sind die Mittelwerte und Standardabweichungen der berücksichtigten Variablen abgebildet. Die manifeste Variable Wohlbefinden in der Pause erreicht den höchsten Mittelwert, der niedrigste findet sich beim negativen affektiven Wohlbefinden, gefolgt vom Leistungsdruck. Bei den Korrelationen (Tab. 3) fällt der hohe Zusammenhang (Cohen 1988) von $r=0,56$ zwischen der sozialen Zughörigkeit und der empfundenen Unterstützung auf. Dies zeigt, dass die Schüler_innen, die sich zur Klasse zugehörig erleben, auch viel Unterstützung erfahren bzw. eine stark empfundene Unterstützung mit hoher sozialer Zugehörigkeit einhergeht. Die Variablen des Wohlbefindens, mit Ausnahme des negativen affektiven schulischen Wohlbefindens, zeigen mittlere Korrelationen mit der sozialen Zugehörigkeit und der empfundenen Unterstützung. Bei der Betrachtung der anderen Prädiktorvariablen lassen sich bei Förderung zur Zusammenarbeit noch die höchsten Korrelationen mit den Zielvariablen finden. Bei den verbleibenden Prädiktoren sind nur kleine oder keine Zusammenhänge nachweisbar.

Tab. 2 Mittelwerte und Standardabweichung aller Variablen getrennt nach den Gruppen von SPF

\begin{tabular}{|c|c|c|c|c|c|c|c|c|}
\hline & \multicolumn{2}{|c|}{$\begin{array}{l}\text { Kein SPF } \\
(N=1319)\end{array}$} & \multicolumn{2}{|c|}{$\begin{array}{l}\text { SPF-L } \\
(N=52)\end{array}$} & \multicolumn{2}{|c|}{$\begin{array}{l}\text { SPF-G } \\
(N=28)\end{array}$} & \multicolumn{2}{|c|}{$\begin{array}{l}\text { SPF-E/S } \\
(N=64)\end{array}$} \\
\hline & $M$ & $S D$ & $M$ & $S D$ & $M$ & $S D$ & $M$ & $S D$ \\
\hline \multicolumn{9}{|l|}{ Wohlbefinden ... } \\
\hline Im Unterricht & 3,01 & 0,78 & 2,95 & 0,84 & 3,14 & 0,90 & 2,67 & 0,80 \\
\hline In der Pause & 3,42 & 0,76 & 3,26 & 0,84 & 3,32 & 0,86 & 3,17 & 0,79 \\
\hline \multicolumn{9}{|c|}{ Affektives schulisches Wohlbefinden: } \\
\hline Positiv & 2,90 & 0,59 & 2,80 & 0,75 & 3,02 & 0,49 & 2,79 & 0,63 \\
\hline Negativ & 2,25 & 0,55 & 2,07 & 0,61 & 2,19 & 0,60 & 2,43 & 0,55 \\
\hline Soziale Zugehörigkeit & 2,94 & 0,55 & 2,78 & 0,69 & 2,97 & 0,70 & 2,64 & 0,67 \\
\hline $\begin{array}{l}\text { Empfundene Unter- } \\
\text { stützung }\end{array}$ & 3,23 & 0,59 & 3,00 & 0,70 & 3,08 & 0,68 & 2,88 & 0,69 \\
\hline Leistungsdruck & 2,34 & 0,64 & 2,57 & 0,75 & 2,78 & 0,67 & 2,59 & 0,72 \\
\hline $\begin{array}{l}\text { Förderung zur Zusam- } \\
\text { menarbeit }\end{array}$ & 2,99 & 0,64 & 3,09 & 0,73 & 3,59 & 0,47 & 2,86 & 0,69 \\
\hline
\end{tabular}




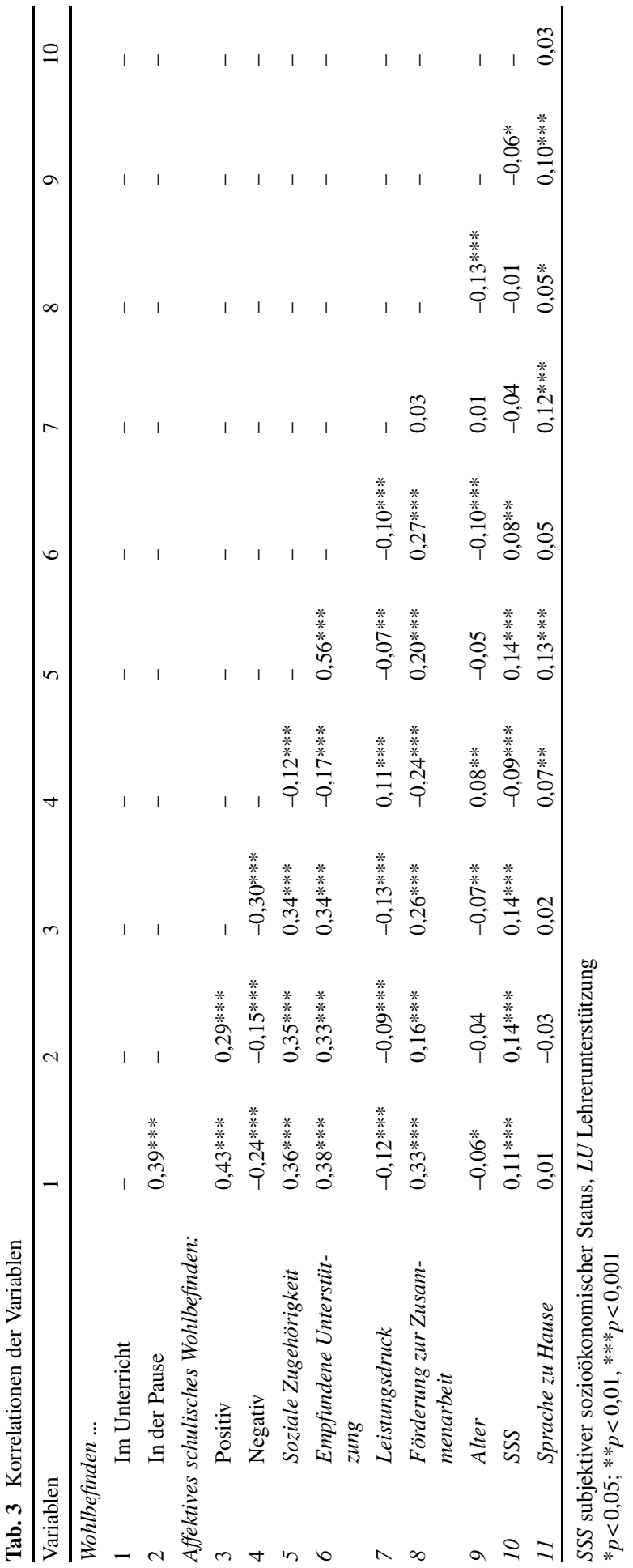


Tab. 4 Vorhersagemodelle für das Wohlbefinden im Unterricht

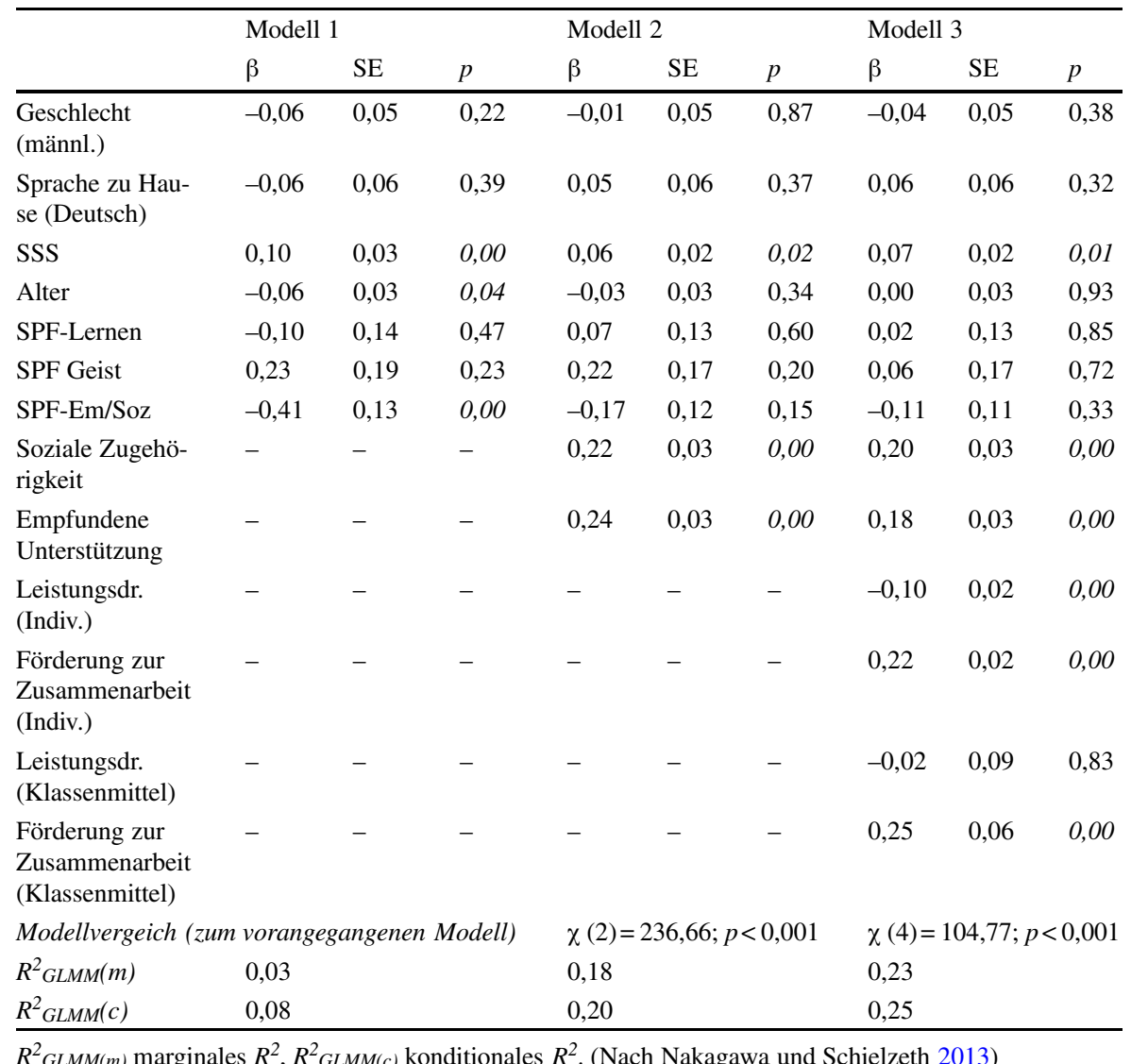

\subsection{Mehrebenenanalyse}

\subsubsection{Modell 1: Einfluss des SPF-Status}

Das jeweils erste Modell soll unter Kontrolle von Geschlecht, Sprache, subjektivem sozioökonomischen Status und dem Alter grundlegend prüfen, inwieweit der Status des Förderschwerpunkts einen bedeutsamen Einfluss auf die sechs Zielvariablen hat. Referenzgruppe beim SPF ist dabei im Folgenden immer die Gruppe der Schüler_innen ohne SPF. Betrachtet man den SPF, zeigt sich, dass der SPF-E/S einen signifikanten Einfluss auf drei der vier Variablen des schulischen Wohnbefindens hat. Sowohl beim Wohlbefinden im Unterricht (Tab. 4), in der Pause (Tab. 5) und beim negativen affektiven Wohlbefinden (Tab. 7) sind bedeutsame Einflüsse des SPF-E/S zu finden. Die $\beta$-Koeffizienten weisen jeweils darauf hin, dass der Status SPF-E/S in diesen drei Zielvariablen ungünstigen Einfluss hat. Interessanterweise wird im Modell des negativen affektiven Wohlbefindens auch SPF-L bedeutsam, jedoch mit einem negativen Koeffizienten (Tab. 6). Dies bedeutet, dass der Status SPF-L hier 
Tab. 5 Vorhersagemodelle für das Wohlbefinden in der Pause

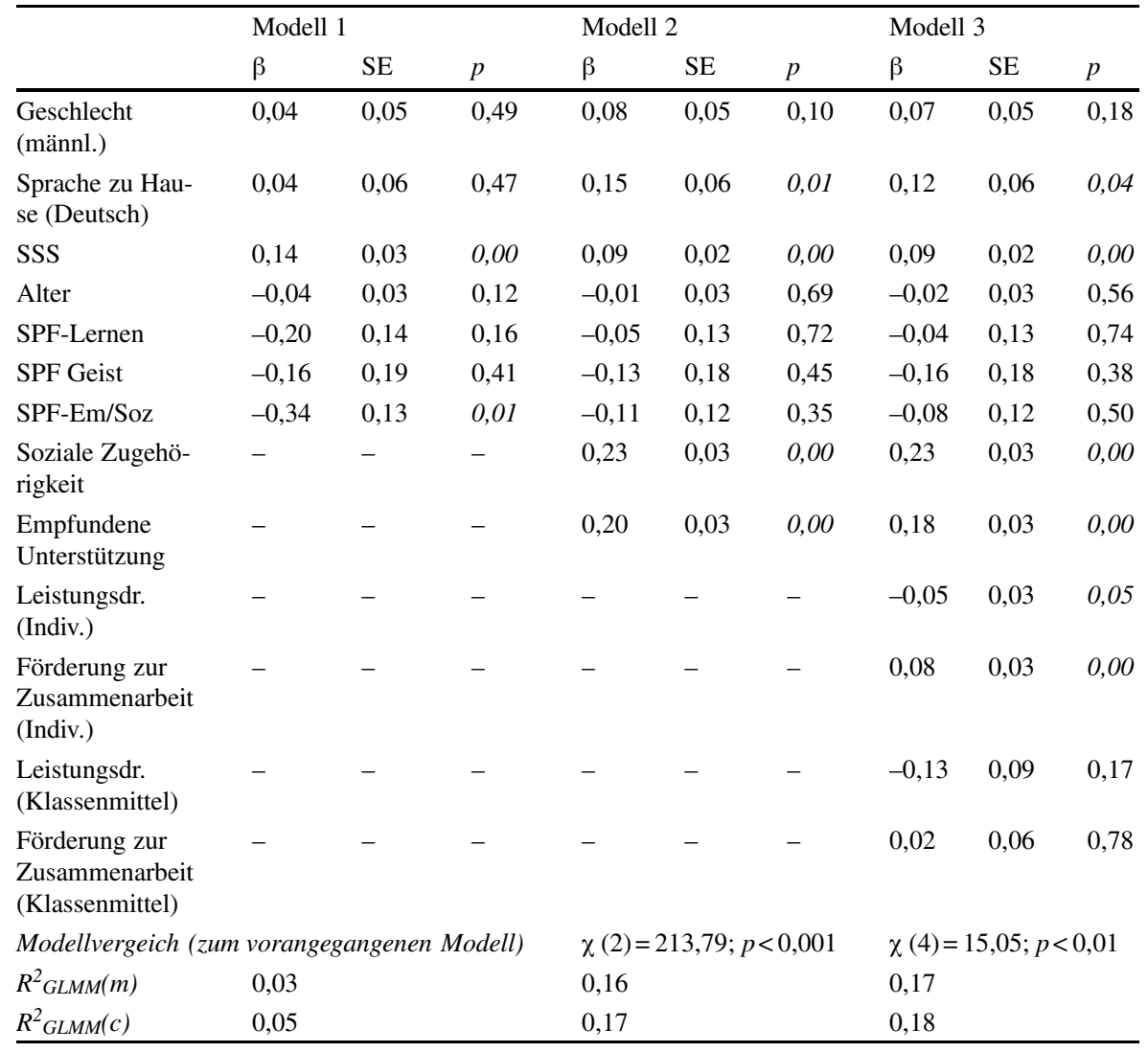

$R^{2} \operatorname{GLMM(m)}$ marginales $R^{2}, R^{2} \operatorname{GLMM(c)}$ konditionales $R^{2}$. (Nach Nakagawa und Schielzeth 2013)

zu einer Reduzierung der negativen Emotionen beiträgt. Von den Kontrollvariablen erweisen sich das Alter und der subjektive sozioökonomische Status als zwei Faktoren, die fast durchgehend signifikant werden, aber mit vergleichsweise geringeren Einflussstärken in den jeweiligen Modellen. Effekte durch das Geschlecht sind beim negativen affektiven Wohlbefinden (Tab. 6) und der empfundenen Unterstützung (Tab. 9) bedeutsam und besagen, dass Jungen über weniger negatives affektives Wohlbefinden, aber auch über weniger empfundene Unterstützung berichten.

Auch in den jeweiligen Modellen mit den beiden abhängigen Variablen wahrgenommene Zugehörigkeit und empfundene Unterstützung zeigen sich abermals negative Einflüsse des Status SPF-E/S auf die Zielvariablen soziale Zugehörigkeit (Tab. 8) und empfundene Unterstützung (Tab. 9). Ebenfalls war hier auch der Status SPF-L bedeutsam. Schüler_innen mit SPF-L erleben weniger soziale Zugehörigkeit und empfinden weniger Unterstützung. Sprechen Schüler_innen überwiegend Deutsch zu Hause, so geht auch dies mit einer geringeren Ausprägung in sozialer Zugehörigkeit und weniger empfundener Unterstützung einher. 
Tab. 6 Vorhersagemodelle für das positive affektive schulische Wohlbefinden

\begin{tabular}{|c|c|c|c|c|c|c|c|c|c|}
\hline & \multicolumn{3}{|c|}{ Modell 1} & \multicolumn{3}{|c|}{ Modell 2} & \multicolumn{3}{|c|}{ Modell 3} \\
\hline & $\beta$ & SE & $p$ & $\beta$ & SE & $p$ & $\beta$ & SE & $p$ \\
\hline $\begin{array}{l}\text { Geschlecht } \\
\text { (männl.) }\end{array}$ & 0,04 & 0,05 & 0,43 & 0,09 & 0,05 & 0,07 & 0,06 & 0,05 & 0,19 \\
\hline $\begin{array}{l}\text { Sprache zu Hau- } \\
\text { se (Deutsch) }\end{array}$ & $-0,06$ & 0,06 & 0,34 & 0,03 & 0,06 & 0,59 & 0,02 & 0,06 & 0,70 \\
\hline SSS & 0,12 & 0,03 & 0,00 & 0,08 & 0,02 & 0,00 & 0,09 & 0,02 & 0,00 \\
\hline Alter & $-0,07$ & 0,03 & 0,01 & $-0,05$ & 0,03 & 0,08 & $-0,04$ & 0,03 & 0,17 \\
\hline SPF-Lernen & $-0,13$ & 0,14 & 0,36 & 0,02 & 0,13 & 0,89 & 0,00 & 0,13 & 0,98 \\
\hline SPF Geist & 0,18 & 0,19 & 0,35 & 0,20 & 0,18 & 0,26 & 0,09 & 0,17 & 0,62 \\
\hline SPF-Em/Soz & $-0,18$ & 0,13 & 0,16 & 0,04 & 0,12 & 0,74 & 0,10 & 0,12 & 0,38 \\
\hline $\begin{array}{l}\text { Soziale Zugehö- } \\
\text { rigkeit }\end{array}$ & - & - & - & 0,20 & 0,03 & 0,00 & 0,18 & 0,03 & 0,00 \\
\hline $\begin{array}{l}\text { Empfundene } \\
\text { Unterstützung }\end{array}$ & - & - & - & 0,22 & 0,03 & 0,00 & 0,17 & 0,03 & 0,00 \\
\hline $\begin{array}{l}\text { Leistungsdr. } \\
\text { (Indiv.) }\end{array}$ & - & - & - & - & - & - & $-0,10$ & 0,02 & 0,00 \\
\hline $\begin{array}{l}\text { Förderung zur } \\
\text { Zusammenarbeit } \\
\text { (Indiv.) }\end{array}$ & - & - & - & - & - & - & 0,20 & 0,03 & 0,00 \\
\hline $\begin{array}{l}\text { Leistungsdr. } \\
\text { (Klassenmittel) }\end{array}$ & - & - & - & - & - & - & 0,02 & 0,09 & 0,86 \\
\hline $\begin{array}{l}\text { Förderung zur } \\
\text { Zusammenarbeit } \\
\text { (Klassenmittel) }\end{array}$ & - & - & - & - & - & - & 0,06 & 0,06 & 0,36 \\
\hline \multicolumn{4}{|c|}{ Modellvergeich (zum vorangegangenen Modell) } & \multicolumn{3}{|c|}{$\chi(2)=199,02 ; p<0,001$} & \multicolumn{3}{|c|}{$\chi(4)=76,14 ; p<0,001$} \\
\hline$R^{2}{ }_{G L M M}(m)$ & \multicolumn{3}{|l|}{0,03} & \multicolumn{3}{|l|}{0,15} & \multicolumn{3}{|l|}{0,19} \\
\hline$R^{2} \operatorname{GLMM}(c)$ & \multicolumn{3}{|l|}{0,05} & \multicolumn{3}{|l|}{0,16} & \multicolumn{3}{|l|}{0,20} \\
\hline
\end{tabular}

$R^{2}{ }_{G L M M(m)}$ marginales $R^{2}, R^{2} \operatorname{GLMM(c)}$ konditionales $R^{2}$. (Nach Nakagawa und Schielzeth 2013)

Die Varianzaufklärung aller sechs Modelle liegt zwischen 3-6\% ( $\left.\mathrm{R}_{\mathrm{GLMM}(\mathrm{m})}\right)$. Die Berücksichtigung des random effect „Klasse“ bei der Varianzaufklärung im $\mathrm{Maß} \mathrm{R}_{\mathrm{GLMM}(\mathrm{c})}$ führt zum Teil zu doppelt so hohen Aufklärungswerten von 5-13\% und deutet die Bedeutsamkeit von Klasseneffekten für die jeweiligen Variablen an.

\subsubsection{Modell 2: Einfluss von sozialer Zugehörigkeit und empfundener Unterstützung}

Im zweiten Modell werden die soziale Zugehörigkeit und die empfundene Unterstützung mit aufgenommen, um deren Einfluss in den Modellen zum affektiven schulischen Wohlbefinden zu überprüfen und deren gegenseitigen Einfluss in den jeweiligen Modellen zu kontrollieren. Zunächst erweisen sich in allen sechs Fällen die zweiten Modelle in einem $\chi^{2}$-Test der Devianzen der Log-Likelihood als signifikant besser als die vorangegangenen Modelle ohne die soziale Zugehörigkeit und die empfundene Unterstützung. Die Varianzaufklärung steigt jeweils stark an, nur beim Modell des negativen affektiven Wohlbefindens fällt dieser Anstieg vergleichsweise gering aus. 
Tab. 7 Vorhersagemodelle für das negative affektive schulische Wohlbefinden

\begin{tabular}{|c|c|c|c|c|c|c|c|c|c|}
\hline & \multicolumn{3}{|c|}{ Modell 1} & \multicolumn{3}{|c|}{ Modell 2} & \multicolumn{3}{|c|}{ Modell 3} \\
\hline & $\beta$ & SE & $p$ & $\beta$ & SE & $p$ & $\beta$ & SE & $p$ \\
\hline $\begin{array}{l}\text { Geschlecht } \\
\text { (männl.) }\end{array}$ & $-0,20$ & 0,05 & 0,00 & $-0,25$ & 0,05 & 0,00 & $-0,22$ & 0,05 & 0,00 \\
\hline $\begin{array}{l}\text { Sprache zu Hau- } \\
\text { se (Deutsch) }\end{array}$ & $-0,09$ & 0,06 & 0,15 & $-0,12$ & 0,06 & 0,05 & $-0,12$ & 0,06 & 0,05 \\
\hline SSS & $-0,08$ & 0,03 & 0,00 & $-0,06$ & 0,03 & 0,03 & $-0,07$ & 0,03 & 0,01 \\
\hline Alter & 0,07 & 0,03 & 0,03 & 0,05 & 0,03 & 0,10 & 0,03 & 0,03 & 0,33 \\
\hline SPF-Lernen & $-0,31$ & 0,14 & 0,02 & $-0,39$ & 0,14 & 0,00 & $-0,36$ & 0,14 & 0,01 \\
\hline SPF Geist & $-0,09$ & 0,19 & 0,64 & $-0,11$ & 0,19 & 0,56 & 0,02 & 0,19 & 0,91 \\
\hline SPF-Em/Soz & 0,39 & 0,13 & 0,00 & 0,29 & 0,13 & 0,02 & 0,23 & 0,12 & 0,06 \\
\hline $\begin{array}{l}\text { Soziale Zugehö- } \\
\text { rigkeit }\end{array}$ & - & - & - & $-0,02$ & 0,03 & 0,48 & $-0,01$ & 0,03 & 0,84 \\
\hline $\begin{array}{l}\text { Empfundene } \\
\text { Unterstützung }\end{array}$ & - & - & - & $-0,18$ & 0,03 & 0,00 & $-0,12$ & 0,03 & 0,00 \\
\hline $\begin{array}{l}\text { Leistungsdr. } \\
\text { (Indiv.) }\end{array}$ & - & - & - & - & - & - & 0,09 & 0,03 & 0,00 \\
\hline $\begin{array}{l}\text { Förderung zur } \\
\text { Zusammenarbeit } \\
\text { (Indiv.) }\end{array}$ & - & - & - & - & - & - & $-0,19$ & 0,03 & 0,00 \\
\hline $\begin{array}{l}\text { Leistungsdr. } \\
\text { (Klassenmittel) }\end{array}$ & - & - & - & - & - & - & 0,03 & 0,11 & 0,80 \\
\hline $\begin{array}{l}\text { Förderung zur } \\
\text { Zusammenarbeit } \\
\text { (Klassenmittel) }\end{array}$ & - & - & - & - & - & - & $-0,19$ & 0,08 & 0,01 \\
\hline \multicolumn{4}{|c|}{ Modellvergeich (zum vorangegangenen Modell) } & \multicolumn{3}{|c|}{$\chi(2)=49,45 ; p<0,001$} & \multicolumn{3}{|c|}{$\chi(4)=70,23 ; p<0,001$} \\
\hline$R^{2} G L M M(m)$ & \multicolumn{3}{|l|}{0,03} & \multicolumn{3}{|l|}{0,06} & \multicolumn{3}{|l|}{0,11} \\
\hline$R^{2}{ }_{G L M M}(c)$ & \multicolumn{3}{|l|}{0,08} & \multicolumn{3}{|l|}{0,10} & \multicolumn{3}{|l|}{0,14} \\
\hline
\end{tabular}

$R^{2} G L M M(m)$ marginales $R^{2}, R^{2}{ }_{G L M M(c)}$ konditionales $R^{2}$. (Nach Nakagawa und Schielzeth 2013)

Die soziale Zugehörigkeit und die empfundene Unterstützung erweisen sich als signifikante Prädiktoren für die Variablen des schulischen Wohlbefindens und gehören zu den stärksten Prädiktoren in ihren jeweiligen Modellen. Lediglich im Fall des negativen affektiven Wohlbefindens erreicht die soziale Zugehörigkeit (Tab. 7) keine Signifikanz, wo hingegen die empfundene Unterstützung (Tab. 9) bedeutsam ist.

Vergleicht man die Veränderung der Einflussstärke von SPF-E/S jeweils zwischen Modell 1 und 2 miteinander, dann fällt auf, dass beim Wohlbefinden im Unterricht und in der Pause, aber auch beim positiven Wohlbefinden der Erklärungswert des SPF-E/S abnimmt. Die empfundene Unterstützung und die soziale Zugehörigkeit klären somit Teile der Einflussstärke des SPF-E/S auf. Beim negativen affektiven Wohlbefinden erweist sich nur der Einfluss der empfundenen Unterstützung (Tab. 7) als bedeutsam. Der Erklärungswert des SPF-E/S nimmt hier im Vergleich zum ersten Modell lediglich geringfügig ab. Somit kann die empfundene Unterstützung zwar negativ erlebte Emotionen verringern, jedoch der Mangel an Unterstützung den 
Tab. 8 Vorhersagemodelle für die soziale Zugehörigkeit

\begin{tabular}{|c|c|c|c|c|c|c|c|c|c|}
\hline & \multicolumn{3}{|c|}{ Modell 1} & \multicolumn{3}{|c|}{ Modell 2} & \multicolumn{3}{|c|}{ Modell 3} \\
\hline & $\beta$ & SE & $p$ & $\beta$ & SE & $p$ & $\beta$ & SE & $p$ \\
\hline $\begin{array}{l}\text { Geschlecht } \\
\text { (männl.) }\end{array}$ & 0,04 & 0,05 & 0,40 & 0,19 & 0,04 & 0,00 & 0,18 & 0,04 & 0,00 \\
\hline $\begin{array}{l}\text { Sprache zu Hau- } \\
\text { se (Deutsch) }\end{array}$ & $-0,27$ & 0,06 & 0,00 & $-0,21$ & 0,05 & 0,00 & $-0,20$ & 0,05 & 0,00 \\
\hline SSS & 0,13 & 0,03 & 0,00 & 0,08 & 0,02 & 0,00 & 0,08 & 0,02 & 0,00 \\
\hline Alter & $-0,06$ & 0,03 & 0,04 & $-0,01$ & 0,02 & 0,60 & 0,00 & 0,03 & 0,97 \\
\hline SPF-Lernen & $-0,32$ & 0,14 & 0,02 & $-0,10$ & 0,12 & 0,36 & $-0,12$ & 0,12 & 0,32 \\
\hline SPF Geist & 0,08 & 0,19 & 0,67 & 0,13 & 0,16 & 0,39 & 0,10 & 0,16 & 0,52 \\
\hline SPF-Em/Soz & $-0,53$ & 0,12 & 0,00 & $-0,25$ & 0,10 & 0,02 & $-0,24$ & 0,10 & 0,02 \\
\hline $\begin{array}{l}\text { Soziale Zugehö- } \\
\text { rigkeit }\end{array}$ & - & - & - & - & - & - & - & - & - \\
\hline $\begin{array}{l}\text { Empfundene } \\
\text { Unterstützung }\end{array}$ & - & - & - & 0,54 & 0,02 & 0,00 & 0,53 & 0,02 & 0,00 \\
\hline $\begin{array}{l}\text { Leistungsdr. } \\
\text { (Indiv.) }\end{array}$ & - & - & - & - & - & - & $-0,03$ & 0,02 & 0,17 \\
\hline $\begin{array}{l}\text { Förderung zur } \\
\text { Zusammenarbeit } \\
\text { (Indiv.) }\end{array}$ & - & - & - & - & - & - & 0,04 & 0,02 & 0,08 \\
\hline $\begin{array}{l}\text { Leistungsdr. } \\
\text { (Klassenmittel) }\end{array}$ & - & - & - & - & - & - & 0,08 & 0,09 & 0,41 \\
\hline $\begin{array}{l}\text { Förderung zur } \\
\text { Zusammenarbeit } \\
\text { (Klassenmittel) }\end{array}$ & - & - & - & - & - & - & 0,07 & 0,06 & 0,30 \\
\hline \multicolumn{4}{|c|}{ Modellvergeich (zum vorangegangenen Modell) } & \multicolumn{3}{|c|}{$\chi(2)=498,27 ; p<0,001$} & \multicolumn{3}{|c|}{$\chi(4)=6,86 ; p=0,14$} \\
\hline$R^{2} G L M M(m)$ & \multicolumn{3}{|l|}{0,05} & \multicolumn{3}{|l|}{0,33} & \multicolumn{3}{|l|}{0,33} \\
\hline$R^{2}{ }_{G L M M}(c)$ & \multicolumn{3}{|l|}{0,11} & \multicolumn{3}{|l|}{0,35} & \multicolumn{3}{|l|}{0,36} \\
\hline
\end{tabular}

Einfluss des SPF-E/S (bzw. den schützenden Effekt des SPF-L) nur geringfügig erklären.

Wie sich schon durch die erwähnte hohe Korrelation zwischen sozialer Zugehörigkeit und empfundener Unterstützung zeigt, so führt auch deren gegenseitiger Einfluss im Modell 2 zu einer Erhöhung des Erklärungswerts der jeweiligen Modelle. Im Modell der sozialen Zugehörigkeit (Tab. 8) trägt die empfundene Unterstützung zu einem großen Sprung in der Varianzaufklärung bei. Im Vergleich zu Modell 1 halbiert sich der Einfluss des SPF-E/S, und der des Status SPF-L wird unbedeutend. Durch die Aufnahme von empfundener Unterstützung wird ein Teil ungeklärter Varianz des Faktors Geschlechts erklärt und somit dessen Vorhersagebeitrag für die soziale Zugehörigkeit erhöht (Suppressionseffekt). Betrachtet man das Modell der empfundenen Unterstützung (Tab. 9), so trägt hier wiederum die soziale Zugehörigkeit in deutlichem Maße zur Varianzaufklärung bei. Sie reduziert den Einfluss von SPF-E/S ebenso um etwa die Hälfte und erklärt Effekte der Sprache und des subjektiven sozioökonomischen Status. 
Tab. 9 Vorhersagemodelle für die empfundene Unterstützung

\begin{tabular}{|c|c|c|c|c|c|c|c|c|c|}
\hline & \multicolumn{3}{|c|}{ Modell 1} & \multicolumn{3}{|c|}{ Modell 2} & \multicolumn{3}{|c|}{ Modell 3} \\
\hline & $\beta$ & SE & $p$ & $\beta$ & SE & $p$ & $\beta$ & SE & $p$ \\
\hline $\begin{array}{l}\text { Geschlecht } \\
\text { (männl.) }\end{array}$ & $-0,27$ & 0,05 & 0,00 & $-0,29$ & 0,04 & 0,00 & $-0,30$ & 0,04 & 0,00 \\
\hline $\begin{array}{l}\text { Sprache zu Hau- } \\
\text { se (Deutsch) }\end{array}$ & $-0,14$ & 0,06 & 0,02 & 0,01 & 0,05 & 0,81 & 0,00 & 0,05 & 0,99 \\
\hline SSS & 0,09 & 0,03 & 0,00 & 0,02 & 0,02 & 0,25 & 0,03 & 0,02 & 0,16 \\
\hline Alter & $-0,10$ & 0,03 & 0,00 & $-0,06$ & 0,02 & 0,02 & $-0,06$ & 0,03 & 0,03 \\
\hline SPF-Lernen & $-0,39$ & 0,14 & 0,00 & $-0,22$ & 0,11 & 0,05 & $-0,23$ & 0,11 & 0,04 \\
\hline SPF Geist & $-0,12$ & 0,18 & 0,51 & $-0,17$ & 0,16 & 0,26 & $-0,27$ & 0,15 & 0,08 \\
\hline SPF-Em/Soz & $-0,52$ & 0,12 & 0,00 & $-0,23$ & 0,10 & 0,03 & $-0,18$ & 0,10 & 0,08 \\
\hline $\begin{array}{l}\text { Soziale Zugehö- } \\
\text { rigkeit }\end{array}$ & - & - & - & 0,54 & 0,02 & 0,00 & 0,50 & 0,02 & 0,00 \\
\hline $\begin{array}{l}\text { Empfundene } \\
\text { Unterstützung }\end{array}$ & - & - & - & - & - & - & - & - & - \\
\hline $\begin{array}{l}\text { Leistungsdr. } \\
\text { (Indiv.) }\end{array}$ & - & - & - & - & - & - & $-0,05$ & 0,02 & 0,02 \\
\hline $\begin{array}{l}\text { Förderung zur } \\
\text { Zusammenarbeit } \\
\text { (Indiv.) }\end{array}$ & - & - & - & - & - & - & 0,16 & 0,02 & 0,00 \\
\hline $\begin{array}{l}\text { Leistungsdr. } \\
\text { (Klassenmittel) }\end{array}$ & - & - & - & - & - & - & $-0,16$ & 0,09 & 0,09 \\
\hline $\begin{array}{l}\text { Förderung zur } \\
\text { Zusammenarbeit } \\
\text { (Klassenmittel) }\end{array}$ & - & - & - & - & - & - & 0,10 & 0,06 & 0,12 \\
\hline \multicolumn{4}{|c|}{ Modellvergeich (zum vorangegangenen Modell) } & \multicolumn{3}{|c|}{$\chi(2)=496,52 ; p<0,001$} & \multicolumn{3}{|c|}{$\chi(4)=673,80 ; p<0,001$} \\
\hline$R^{2} G L M M(m)$ & \multicolumn{3}{|l|}{0,06} & \multicolumn{3}{|l|}{0,33} & \multicolumn{3}{|l|}{0,36} \\
\hline$R^{2} \operatorname{GLMM}(c)$ & \multicolumn{3}{|l|}{0,13} & \multicolumn{3}{|l|}{0,36} & \multicolumn{3}{|l|}{0,39} \\
\hline
\end{tabular}

$R^{2}{ }_{\operatorname{GLMM}(m)}$ marginales $R^{2}, R^{2}{ }_{G L M M(c)}$ konditionales $R^{2}$. (Nach Nakagawa und Schielzeth 2013)

\subsubsection{Modell 3: Einfluss von Leistungsdruck und der Förderung zur Zusammenarbeit}

Im dritten Schritt werden den Modellen die Konstrukte Leistungsdruck und Förderung zur Zusammenarbeit durch die Lehrkräfte jeweils als individueller Faktor auf der ersten und als aggregierte Variablen auf der zweiten Modellebene hinzugefügt. Im Vergleich zu den vorangegangenen Modellen erweisen sich die neuen Modelle wiederum als erklärungsstärker, mit Ausnahme des Modells der sozialen Zugehörigkeit (Tab. 8). Die Zuwächse in der Varianzaufklärung fallen jedoch zum vorangegangenen Zuwachs zwischen Modell 1 und 2 deutlich geringer aus. Im Vergleich zu Modell 2 gibt es in den zuvor schon aufgenommenen Variablen keine deutlichen Veränderungen. Dabei werden die neuen Faktoren Leistungsdruck und Förderung zur Zusammenarbeit vorwiegend auf der Individualebene signifikant.

Der individuell empfundene Leistungsdruck ist zwar für alle vier Variablen aus dem schulischen Wohlbefinden ein statistisch bedeutsamer Prädiktor, die $\beta$-Koeffizienten gehören dabei jedoch jeweils zu den schwächsten Prädiktoren. Die indi- 
viduell wahrgenommene Förderung zur Zusammenarbeit erweist sich dagegen für einige Aspekte des schulischen Wohlbefindens als stärkerer Prädiktor. Besonders beim schulischen Wohlbefinden im Unterricht (Tab. 4) und dem positiven affektiven Wohlbefinden (Tab. 6) zeigt sich, dass die Förderung zur Zusammenarbeit einen günstigen Einfluss auf der Individual- und der Klassenebene hat.

Für die soziale Zugehörigkeit (Tab. 8) sind dagegen weder der Leistungsdruck noch die wahrgenommene Förderung zur Zusammenarbeit geeignete Prädiktoren. Für die empfundene Unterstützung (Tab. 9) erreicht der Prädiktor Leistungsdruck auf Individualebene statistische Signifikanz und stellt den kleinsten der bedeutsamen $\beta$-Koeffizienten in diesem Modell dar. Für die wahrgenommene Förderung zur Zusammenarbeit (Indiv.) kann jedoch ein positiver Einfluss auf die empfundene Unterstützung belegt werden.

\section{Diskussion}

In der vorliegenden Studie wurden die soziale Zugehörigkeit, das schulische Wohlbefinden und die Unterstützung von Schüler_innen der 7.-10. Klasse im inklusiven Unterricht in Berliner und Brandenburger Schulen untersucht. Es wurde näher betrachtet, inwiefern das affektive schulische Wohlbefinden, die soziale Zugehörigkeit und die empfundene Unterstützung vom sonderpädagogischen Förderstatus abhängen. Dabei wurden die sonderpädagogischen Förderschwerpunkte in den Bereichen Lernen, geistige Entwicklung und emotionale und soziale Entwicklung für die Auswertungen differenziert und es wurde untersucht, inwiefern sich Gruppenunterschiede aufgrund des SPF durch Prädiktoren wie die soziale Zugehörigkeit, empfundene Unterstützung, Leistungsdruck und Förderung zur Zusammenarbeit durch die Lehrkräfte erklären lassen.

\subsection{Schulisches Wohlbefinden}

Es zeigt sich, dass sich nicht generell alle Gruppen von Schüler_innen mit SPF in ihren Klassen weniger wohlfühlen als ihre Mitschüler_innen ohne SPF. So erleben Schüler_innen mit Förderstatus geistige Entwicklung bzw. Lernen ein vergleichbares Maß an affektivem schulischem Wohlbefinden wie Schüler_innen ohne SPF. Jedoch weisen die Schüler_innen mit SPF-E/S, mit Ausnahme des positiven affektiven schulischen Wohlbefindens, bei den anderen drei Variablen des Wohlbefindens geringere Werte auf. Auch Venetz et al. (2010) fanden heraus, dass Lernende mit Verhaltensauffälligkeiten im Unterricht stärker negativ aktiviert sind, jedoch konnte in der vorliegenden Untersuchung zusätzlich ein geringeres Wohlbefinden im Unterricht und in den Pausen für diese Gruppe von Schüler_innen nachgewiesen werden. Auch für den Status SPF-L wurde bei einer Variablen zum Wohlbefinden ein bedeutsamer Einfluss nachgewiesen. Überraschenderweise erleben Schüler_innen mit SPF-L weniger negative Emotionen in der Schule. Eventuell könnte es daran liegen, dass Schüler_innen mit SPF-L einen höheren Unterstützungsbedarf beim Lernen haben und im Unterricht mehr Aufmerksamkeit von den Lehrkräften erhalten, wodurch sie eventuell auch frühzeitiger Unterstützung erhalten, wenn sie stark negative 
Emotionen erleben. Dem widerspricht jedoch, dass in diesem Fall auch geringere negative Emotionen für die Schüler_innen mit SPF-G zu erwarten wären, was hier nicht der Fall ist.

\subsection{Soziale Zugehörigkeit und empfundene Unterstützung}

Auch bei der sozialen Zugehörigkeit und der empfundenen Unterstützung sind es die Schüler_innen mit SPF-E/S, die ihre Situation in diesen sozialen Aspekten schlechter einschätzen als die Schüler_innen ohne diagnostizierten Förderbedarf. Dies bestätigt das schon zuvor in anderen Untersuchungen gefundene erhöhte Ausgrenzungsrisiko für Schüler_innen mit sozial-emotionalen Problemen (Bierman et al. 1993; Chang 2004; Gottlieb et al. 1986) und zeigt, dass sich diese Gruppe von Schüler_innen nicht nur weniger wohl in der Schule fühlen, sondern sich ebenso weniger unterstützt und zugehörig zur Klasse erleben als ihre Mitschüler_innen ohne SPF. Der Förderschwerpunkt Lernen steht aber auch mit geringerer empfundener Unterstützung in Verbindung, womit sich die geringere Unterstützung von lernschwachen Schüler_innen in Grundschulen (Kulawiak und Wilbert 2015; Wenz-Gross und Siperstein 1998) auch in der Sekundarstufe fortzusetzen scheint. Für den SPF-G konnte hingegen kein Einfluss auf die soziale Zugehörigkeit und die empfundene Unterstützung nachgewiesen werden, und in der hier untersuchten Kohorte weicht damit das soziale Erleben von Schüler_innen mit SPF-G nicht von dem der Schüler_innen ohne SPF ab. Wie Untersuchungen, wie die von Nordström (2011), zeigen, ist aber zu erwarten, dass Schüler_innen mit kognitiver Beeinträchtigung quantitativ gemessen weniger Interaktionen, besonders mit ihren Mitschüler_innen ohne Beeinträchtigung, haben. Vermutlich haben sie aber ein geringes Anspruchsniveau hinsichtlich der Teilnahme an sozialen Aktivitäten mit Gleichaltrigen (Carver 2000), weshalb sie, trotz weniger Interaktionen mit Mitschüler_innen subjektiv eine vergleichbare Zufriedenheit bezüglich ihrer sozialen Zugehörigkeit und der Unterstützung haben.

Die Analysen zeigen außerdem, dass das Maß an wahrgenommener Unterstützung und die soziale Zugehörigkeit mit dem Wohlbefinden zusammenhängen (eine Ausnahme bildet hier der Zusammenhang zwischen sozialer Zugehörigkeit und negativem affektivem Wohlbefinden). Schon in anderen Untersuchungen wurde belegt, dass die Zugehörigkeit und die Art der sozialen Interaktionen wesentlich zum Wohlbefinden beitragen (Eder 1995; Krappmann und Oswald 1995). Daher können in der geringeren sozialen Zugehörigkeit und geringeren Unterstützung wichtige Gründe gesehen werden, warum sich Schüler_innen mit SPF-E/S im Unterricht und in den Pausen weniger wohl fühlen. Allerdings können beide Konstrukte das geringere Wohlbefinden der Schüler_innen mit SPF-E/S nur teilweise erklären.

\subsection{Leistungsdruck und Förderung zur Zusammenarbeit}

Der Leistungsdruck erweist sich in dieser Untersuchung als kein besonders starker Faktor. Es findet sich nur ein verhältnismäßig kleiner Einfluss auf die Variablen des affektiven schulischen Wohlbefindens. Dies weicht ab von den Ergebnissen von Hascher (2004), in denen sich der Leistungsdruck als ein wichtiger Prädiktor zur Vorhersage der Teilkomponenten des Wohlbefindens erwies. Es könnte dabei 
relevant sein, welchen Ursprung die Schüler_innen für ihren Leistungsdruck wahrnehmen. Kommt dieser Druck von ihnen selbst, aufgrund des eigenen Anspruchs an die Leistung, oder wird der Druck von Eltern bzw. Lehrkräften auf sie ausgeübt? Eventuell wäre Letzteres etwa im Sinne der Selbstbestimmungstheorie (Deci und Ryan 2000) schlechter für die Motivation und das emotionale Erleben. Es wäre also für zukünftige Untersuchungen wichtig, auch den wahrgenommenen Ursprung des empfundenen Leistungsdrucks zu klären. Ebenso ist zu beachten, dass der Intraklassenkorrelationskoeffizient beim Leistungsdruck, und somit der Varianzanteil, der durch die Klassenebene erklärt werden kann, recht gering ausfällt. Dies wird ursächlich dafür sein, warum dieses Konstrukt auf Klassenebene keinen bedeutsamen Erklärungswert zeigt. Inhaltlich überrascht, dass zwischen Schüler_innen verschiedener Klassen (aus unterschiedlichen Schulen) der Unterschied im empfundenen Leistungsdruck so gering ist. Zusammen mit den geringen Korrelationswerten zu anderen Konstrukten (besonders denen des Wohlbefindens) sollte die Validität der Skala Leistungsdruck hinterfragt werden, weshalb eine Revision des Instruments für zukünftige Untersuchungen angeregt wird.

Wenn die Lehrkräfte so wahrgenommen werden, dass sie die Schüler_innen zur gegenseitigen Unterstützung und Zusammenarbeit anhalten, dann steht dies allgemein in Verbindung mit besserem affektivem schulischem Wohlbefinden und mehr empfundener Unterstützung. Es zeigt sich auch ein Zusammenhang mit der sozialen Zugehörigkeit, wobei in der Mehrebenenanalyse dieser Faktor nicht bedeutsam wird. Die Frage nach der Kausalität der jeweiligen Einflüsse bleibt hierbei offen. Es erscheint plausibel anzunehmen, dass Schüler_innen, die sich im Unterricht weniger wohl und unterstützt fühlen, auch kritischer mit ihren Lehrkräften sind und höhere Erwartungen bezüglich Unterstützung zur Einbindung haben. So kommt Schweer (1997) zu dem Schluss, dass für Schüler_innen beim Beurteilen von realem Verhalten der Lehrkräfte oft ein Idealbild als Grundlage dient. Das Verhalten der Lehrkräfte bezüglich ihrer Förderung von gegenseitiger Unterstützung und Wertschätzung gegenüber Diversität sollte auch zukünftig nicht nur in der multikulturellen Inklusionsforschung berücksichtigt werden. So bieten sich über diese Verhaltensweisen der Lehrkräfte potenziell gute Ansatzpunkte für die Förderung des psycho-sozialen Erlebens von Schüler_innen im gemeinsamen Unterricht.

Insgesamt sollte bei der Interpretation der Ergebnisse für die Förderung zur $\mathrm{Zu}$ sammenarbeit durch die Lehrkräfte und den Leistungsdruck berücksichtigt werden, dass hier überwiegend die Individualvariablen die bedeutsamen Effekte erreichen. Eine mögliche Erklärung könnte sein, dass es zwar eine Varianz zwischen den Klassen gibt, diese aber recht gering ausfällt. Inhaltlich deutet dies an, dass entscheidender ist, dass die Förderung zur Zusammenarbeit generell als hoch wahrgenommen wird, und diese Wahrnehmung nicht nur im Vergleich zu den Mitschüler_innen in der Klasse hoch ausfällt. Beim Wohlbefinden im Unterricht und dem negativen affektiven Wohlbefinden hingegen kann zusätzlich dazu auch der relative Unterschied zu den Mitschüler_innen in der wahrgenommenen Förderung zur Zusammenarbeit einen günstigen Einfluss haben. Möglicherweise hat hier eine stärkere individuelle Unterstützung durch die Lehrkräfte einen zusätzlichen förderlichen Einfluss auf das Wohlbefinden im Unterricht (bzw. senkt das negative affektive Wohlbefinden), zum 
Beispiel, wenn einige Schüler_innen in der jeweiligen Klasse mehr Unterstützung bei Gruppenprozessen von den Lehrkräften erfahren.

\subsection{Schlussfolgerungen}

In der vorliegenden Untersuchung wurden Unterschiede im affektiven schulischen Wohlbefinden, der sozialen Zugehörigkeit und der wahrgenommenen Unterstützung besonders zwischen Schüler_innen mit SPF-E/S und denen ohne SPF in der 7.-10. Klassenstufe gefunden. Dies sollte im Rahmen des inklusiven Unterrichts eine stärkere Aufmerksamkeit auf diese Gruppe von Schüler_innen lenken, die sich in diesen Klassen weniger wohl und aufgenommen fühlen. Lehrkräfte sollten verstärkt die Zusammenarbeit und Unterstützung zwischen den Schüler_innen fördern. Dies betrifft aber nicht nur die Schüler_innen mit SPF-L, deren Förderstatus sich mit geringerer empfundener Unterstützung verbunden zeigt, sondern es sollte verstärkt auch die Kooperation mit sozial-emotional auffälligeren Schüler_innen gefördert werden. So konnten Pepler et al. (1998) nachweisen, dass das Erfahren von prosozialem Verhalten zu einer Verstärkung von prosozialen Verhaltensweisen bei verhaltensauffälligen Schüler_innen führen kann, Ablehnung hingegen verstärkt eher negative Verhaltensweisen. Diese positiven sozialen Erfahrungen könnten dann, neben den positiven Effekten auf das psycho-soziale Wohlbefinden, auch das prosoziale Verhalten bei Schüler_innen mit SPF-E/S verstärken.

In den Gesprächen mit den Lehrkräften wurde wiederholt erwähnt, dass sie vielfältige Wege kennen, wie sie mit Leistungsheterogenität umgehen können und sie diese, aufgrund der Planbarkeit bei der Unterrichtsvorbereitung, als weniger problematisch erleben. Soziale und emotionale Probleme hingegen stellen eine sehr große Herausforderung für sie dar, auf die sie sich in Aus- und Weiterbildung weniger gut vorbereitet fühlen. Daher erscheint gerade in diesem Bereich Verbesserungsbedarf in der Aus- und Fortbildung von deutschen Lehrkräften zu bestehen.

\subsection{Einschränkungen und Implikationen für zukünftige Untersuchungen}

Abschließend sei auf einige Einschränkungen der Untersuchung hingewiesen. Die Fallzahl an Schüler_innen für die einzelnen Förderschwerpunkte ist relativ gering, wenn dies auch anhand des Anteils von Schüler_innen mit SPF so zu erwarten war. Daher können andere Schwerpunkte, z.B. Autismus oder körperliche und motorische Entwicklung, nicht gesondert betrachtet werden. Durch die geringen Fallzahlen in den berücksichtigen Förderschwerpunkten können kleine Effekte unentdeckt geblieben sein. Für zukünftige Untersuchungen ist daher eine vorherige Festlegung der notwendigen Mindestgröße der jeweiligen SPF Gruppen durch Power-Analysen empfohlen (Rost 2013). Da in der Mehrebenenanalyse die Faktoren nicht als latente Konstrukte modelliert werden konnten, kann es durch den Verlust an Varianz zu Verzerrungen gekommen sein. Eine Mehrebenen-Strukturgleichungsanalyse wäre für weiterführenden Untersuchungen als strukturprüfendes Verfahren empfehlenswert.

Es ist auch zu bedenken, dass die Teilnahme an der Befragung von der Zustimmung der Schüler_innen und deren Eltern abhängig war. Hier kann ein möglicher 
Selektionseffekt nicht ausgeschlossen werden, bei dem z. B. besonders unzufriedene Schüler_innen oder solche aus weniger engagierten Familien überproportional häufig nicht an der Befragung teilnahmen.

Schüler_innen mit SPF-G zeigen ein ähnlich hohes schulisches Wohlbefinden, Gefühl der Zugehörigkeit und der Unterstützung wie Schüler_innen ohne SPF, jedoch ist kritisch zu bemerken, dass durch die häufige Unterstützung durch schulisches Personal eine stärkere Verzerrung im Sinne der sozialen Erwünschtheit vorliegen könnte. Vom Lehrpersonal wurde gelegentlich angemerkt, dass für die Schüler_innen mit SPF-G einige Fragen schwer zu verstehen seien. Für zukünftige Erhebungen sollten die Instrumente daraufhin geprüft werden, ob eine weitere Vereinfachung (z.B. mit Symbolen wie Smileys als Bewertungsstufen) möglich ist. Daher wird angeregt, dass in zukünftigen Untersuchungen versucht wird, besonders die Ergebnisse für SPF-G mit größeren Stichproben und revidierten Instrumenten (speziell die Skala Leistungsdruck) zu replizieren.

Funding Open Access funding provided by Projekt DEAL.

Open Access Dieser Artikel wird unter der Creative Commons Namensnennung 4.0 International Lizenz veröffentlicht, welche die Nutzung, Vervielfältigung, Bearbeitung, Verbreitung und Wiedergabe in jeglichem Medium und Format erlaubt, sofern Sie den/die ursprünglichen Autor(en) und die Quelle ordnungsgemäß nennen, einen Link zur Creative Commons Lizenz beifügen und angeben, ob Änderungen vorgenommen wurden.

Die in diesem Artikel enthaltenen Bilder und sonstiges Drittmaterial unterliegen ebenfalls der genannten Creative Commons Lizenz, sofern sich aus der Abbildungslegende nichts anderes ergibt. Sofern das betreffende Material nicht unter der genannten Creative Commons Lizenz steht und die betreffende Handlung nicht nach gesetzlichen Vorschriften erlaubt ist, ist für die oben aufgeführten Weiterverwendungen des Materials die Einwilligung des jeweiligen Rechteinhabers einzuholen.

Weitere Details zur Lizenz entnehmen Sie bitte der Lizenzinformation auf http://creativecommons.org/ licenses/by/4.0/deed.de.

\section{Literatur}

Adler, N., \& Stewart, J. (2007). The macArthur scale of subjective social status. macArthur research network on SES \& health. http://www.macses.ucsf.edu/research/psychosocial/subjective.php. Zugegriffen: 3. Juli 2018.

Arbuckle, J.L. (2017). Amos (version 25.0) [computer program]. Chicago: IBM SPSS.

Avramidis, E. (2010). Social relationships of pupils with special educational needs in the mainstream primary class: peer group membership and peer-assessed social behaviour. European Journal of Special Needs Education, 25(4), 413-429. https://doi.org/10.1080/08856257.2010.513550.

Bakker, J. T. A., Denessen, E., Bosman, A. M. T., Krijger, E.-M., \& Bouts, L. (2007). Sociometric status and self-image of children with specific and general learning disabilities in Dutch general and special education classes. Learning Disability Quarterly, 30(1), 47. https://doi.org/10.2307/30035515.

Barnes, M., \& Harrison, E. (2017). The wellbeing of secondary school pupils with special educational needs. London: Department for Education.

Bates, D., Mächler, M., Bolker, B., \& Walker, S. (2015). Fitting Linear Mixed-Effects Models Using lme4. Journal of Statistical Software, 67(1). https://doi.org/10.18637/jss.v067.i01

Bayram Özdemir, S., \& Stattin, H. (2014). Why and when is ethnic harassment a risk for immigrant adolescents' school adjustment? Understanding the processes and conditions. Journal of Youth and Adolescence, 43(8), 1252-1265. https://doi.org/10.1007/s10964-013-0038-y.

Bierman, K.L., Smoot, D.L., \& Aumiller, K. (1993). Characteristics of aggressive-rejected, aggressive (nonrejected), and rejected (nonaggressive) boys. Child development, 64(1), 139-151. 
Bless, G., \& Mohr, K. (2007). Die Effekte von Sonderunterricht und gemeinsamem Unterricht auf die Entwicklung von Kindern mit Lernbehinderungen. In J. Walter \& F. B. Wember (Hrsg.), Sonderpädagogik des Lernens (S. 375-383). Göttingen: Hogrefe.

Bossaert, G., de Boer, A. A., Frostad, P., Pijl, S. J., \& Petry, K. (2015). Social participation of students with special educational needs in different educational systems. Irish Educational Studies, 34(1), 43-54. https://doi.org/10.1080/03323315.2015.1010703.

Bossaert, G., Colpin, H., Pijl, S.J., \& Petry, K. (2013). Truly included? A literature study focusing on the social dimension of inclusion in education. International Journal of Inclusive Education, 17(1), 60-79. https://doi.org/10.1080/13603116.2011.580464.

Bryan, T., Burstein, K., \& Ergul, C. (2004). The social-emotional side of learning disabilities: a sciencebased presentation of the state of the Art. Learning Disability Quarterly, 27(1), 45-51. https://doi. org/10.2307/1593631.

Cambra, C., \& Silvestre, N. (2003). Students with special educational needs in the inclusive classroom: social integration and self-concept. European Journal of Special Needs Education, 18(2), 197-208. https://doi.org/10.1080/0885625032000078989.

Carver, C.S. (2000). On the continuous calibration of happiness. American Journal on Mental Retardation, 105(5), 336. https://doi.org/10.1352/0895-8017(2000)105〈0336:OTCCOH $\rangle$ 2.0.CO;2.

Chang, L. (2004). The role of classroom norms in contextualizing the relations of children's social behaviors to Peer acceptance. Developmental Psychology, 40(5), 691-702. https://doi.org/10.1037/00121649.40.5.691.

Cohen, J. (1988). Statistical power analysis for the behavioral sciences. Hillsdale: Erlbaum.

Cohen, J., Cohen, P., West, S. G., \& Aiken, L. S. (2003). Applied multiple regression/correlation analysis for the behavioral sciences. Mahwah: Lawrence Erlbaum.

Deci, E., \& Ryan, R.M. (2000). The „what“ and „why“ of goal pursuits: human needs and the self-determination of behavior. Psychological Inquiry, 11(4), 227-268. https://doi.org/10.1207/ S15327965PLI1104_01.

Doubt, L., \& McColl, M.A. (2003). A secondary guy: physically disabled teenagers in secondary schools. Canadian Journal of Occupational Therapy, 70(3), 139-151. https://doi.org/10.1177/ 000841740307000303.

Dumke, D., \& Schäfer, G. (1993). Entwicklung behinderter und nichtbehinderter Schüler in Integrationsklassen: Einstellungen, soziale Beziehungen, Persönlichkeitsmerkmal und Schulleistungen. Weinheim: Deutscher Studien Verlag.

Dumont, M., \& Provost, M. A. (1999). Resilience in Adolescents: Protective Role of Social Support, Coping Strategies, Self-Esteem, and Social Activities on Experience of Stress and Depression. Journal of Youth and Adolescence, 28(2), 343-363. https://doi.org/10.1023/A:1021637011732.

Eder, F. (1995). Das Befinden von Schülerinnen und Schülern in öffentlichen Schulen - Ergebnisse der Repräsentativerhebung. In F. Eder (Hrsg.), Das Befinden von Kindern und Jugendlichen in der Schule (S. 24-168). Innsbruck: StudienVerlag.

Eder, F. (2007). Das Befinden von Kindern und Jugendlichen in der österreichischen Schule. Innsbruck: StudienVerlag.

Enders, C.K., \& Tofighi, D. (2007). Centering predictor variables in cross-sectional multilevel models: a new look at an old issue. Psychological Methods, 12(2), 121-138. https://doi.org/10.1037/1082989X.12.2.121.

Estell, D.B., Jones, M.H., Pearl, R., Van Acker, R., Farmer, T.W., \& Rodkin, P.C. (2008). Peer groups, popularity, and social preference. Journal of Learning Disabilities, 41(1), 5-14. https://doi.org/10. 1177/0022219407310993.

Frederickson, N.L., \& Furnham, A.F. (2004). Peer-assessed behavioural characteristics and sociometric rejection: differences between pupils who have moderate learning difficulties and their mainstream peers. British Journal of Educational Psychology, 74(3), 391-410. https://doi.org/10.1348/ 0007099041552305.

Garrote, G. (2017). Relationship between the social participation and social skills of pupils with an intellectual disability: a study in inclusive classrooms. Frontline Learning Research, 5(1), 1-15. https:// doi.org/10.14786/flr.v5i1.266.

Gaspar, T., Bilimória, H., Albergaria, F., \& Matos, M. G. (2016). Children with special education needs and subjective well-being: social and personal influence. International Journal of Disability, Development and Education, 63(5), 500-513. https://doi.org/10.1080/1034912X.2016.1144873.

Gasser, L., Grütter, J., Torchetti, L., \& Buholzer, A. (2017). Competitive classroom norms and exclusion of children with academic and behavior difficulties. Journal of Applied Developmental Psychology, 49, 1-11. https://doi.org/10.1016/j.appdev.2016.12.002. 
Gebhardt, M., \& Heimlich, U. (2018). Inklusion und Bildung. In R. Tippelt \& B. Schmidt-Hertha (Hrsg.), Handbuch Bildungsforschung (S. 1241-1260). Wiesbaden: Springer VS. https://doi.org/10.1007/ 978-3-531-19981-8_55.

Gottlieb, B.W., Gottlieb, J., Berkell, D., \& Levy, L. (1986). Sociometric status and solitary play of LD boys and girls. Journal of Learning Disabilities, 19(10), 619-622. https://doi.org/10.1177/ 002221948601901008.

Green, N., \& Green, K. (2005). Kooperatives Lernen im Klassenraum und im Kollegium: Das Trainingsbuch. Seelze: Klett.

Grosche, M. (2015). Was ist Inklusion? In P. Kuhl, P. Stanat, B. Lütje-Klose, C. Gresch, H. A. Pant \& M. Prenzel (Hrsg.), Inklusion von Schülerinnen und Schülern mit sonderpädagogischem Förderbedarf in Schulleistungserhebungen (S. 17-39). Wiesbaden: Springer. https://doi.org/10.1007/978-3658-06604-8_1.

Gruehn, S. (1995). Vereinbarkeit kognitiver und nichtkognitiver Ziele im Unterricht. Zeitschrift für Pädagogik, 41(4), 531-553.

Grüning, E. (2011). Emotionales Wohlbefinden von Kinde rn und Jugendlichen mit geistiger Behinderung im schulischen Kontext unter inklusiven und segregativen Bedingungen. Heilpädagogische Forschung, 37(1), 13-22.

Haeberlin, U., Bless, G., Moser, U., \& Klaghofer, R. (1991). Die Integration von Lernbehinderten. Versuche, Theorien, Forschungen, Enttäuschungen, Hoffnungen. Bern: Haupt.

Haeberlin, U., Bless, G., Moser, U., \& Klaghofer, R. (1999). Die Integration von Lernbehinderten: Versuche, Theorien, Forschungen, Enttäuschungen, Hoffnungen. Bern: Haupt.

Haeberlin, U., Moser, U., Bless, G., \& Klaghofer, R. (1989). Integration in die Schulklasse. Fragebogen zur Erfassung von Dimensionen der Integration von Schülern FDI 4-6. Bern: Haupt Verlag.

Hagenauer, G. (2011). Lernfreude in der Schule. Münster: Waxmann.

Hascher, T. (2004). Wohlbefinden in der Schule. Münster: Waxmann.

Hascher, T., \& Lobsang, K. (2004). Das Wohlbefinden von SchülerInnen - Faktoren, die es stärken und solche, die es schwächen. In Schule positiv erleben: Ergebnisse und Erkenntnisse zum Wohlbefinden von Schülerinnen und Schülern (S. 203-228). Bern: Haupt.

Henke, T., Bogda, K., Lambrecht, J., Bosse, S., Koch, H., Maaz, K., et al. (2017). Will you be my friend? A multilevel network analysis of friendships of students with and without special educational needs backgrounds in inclusive classrooms. Zeitschrift für Erziehungswissenschaft, 20(3), 449-474. https:// doi.org/10.1007/s11618-017-0767-x.

Hoebel, J., Müters, S., Kuntz, B., Lange, C., \& Lampert, T. (2015). Messung des subjektiven sozialen Status in der Gesundheitsforschung mit einer deutschen Version der MacArthur Scale. Bundesgesundheitsblatt - Gesundheitsforschung - Gesundheitsschutz, 58(7), 749-757. https://doi.org/10.1007/ s00103-015-2166-X.

Huber, C. (2008). Jenseits des Modellversuchs: Soziale Integration von Schülern mit sonderpädagogischem Förderbedarf im Gemeinsamen Unterricht - Eine Evaluationsstudie. Heilpädagogische Forschung, 1, $2-14$.

Huber, C., \& Wilbert, J. (2012). Soziale Ausgrenzung von Schülern mit sonderpädagogischem Förderbedarf und niedrigen Schulleistungen im gemeinsamen Unterricht. Empirische Sonderpädagogik, 4, $147-165$.

Humphrey, N., \& Symes, W. (2010). Perceptions of social support and experience of bullying among pupils with autistic spectrum disorders in mainstream secondary schools. European Journal of Special Needs Education, 25(1), 77-91. https://doi.org/10.1080/08856250903450855.

IBM Corp (2017). IBM SPSS statistics for windows, version 25.0. Armonk: IBM Corp.

Kemp, C., \& Carter, M. (2002). The social skills and social status of mainstreamed students with intellectual disabilities. Educational Psychology, 22(4), 391-411. https://doi.org/10.1080/014434102200000 3097.

Killen, M., \& Stangor, C. (2001). Children's social reasoning about inclusion and exclusion in gender and race peer group contexts. Child Development, 72(1), 174-186. https://doi.org/10.1111/1467-8624. 00272.

Klicpera, C. (2007). Erfahrungen von Eltern und Schulaufsicht mit dem Elternwahlrecht in der Entscheidung über den Unterrichtsort. Münster: LIT.

Kocaj, A., Kuhl, P., Kroth, A.J., Pant, H. A., \& Stanat, P. (2014). Wo lernen Kinder mit sonderpädagogischem Förderbedarf besser? Ein Vergleich schulischer Kompetenzen zwischen Regel- und Förderschulen in der Primarstufe. KZfSS Kölner Zeitschrift für Soziologie und Sozialpsychologie, 66(2), 165-191. https://doi.org/10.1007/s11577-014-0253-X. 
Koster, M., Nakken, H., Pijl, S. J., \& van Houten, E. (2009). Being part of the peer group: a literature study focusing on the social dimension of inclusion in education. International Journal of Inclusive Education, 13(2), 117-140. https://doi.org/10.1080/13603110701284680.

Koster, M., Pijl, S. J., Nakken, H., \& Van Houten, E. (2010). Social participation of students with special needs in regular primary education in the Netherlands. International Journal of Disability, Development and Education, 57(1), 59-75. https://doi.org/10.1080/10349120903537905.

Krappmann, L., \& Oswald, H. (1995). Alltag der Schulkinder. München: Juventa.

Krawinkel, S., Südkamp, A. S., \& Tröster, H. (2017). Soziale Partizipation in inklusiven Grundschulklassen: Bedeutung von Klassen- und Lehrkraftmerkmalen. Empirische Sonderpädagogik, 3, 277-295.

Krull, J., Wilbert, J., \& Hennemann, T. (2014a). Soziale Ausgrenzung von Erstklässlerinnen und Erstklässlern mit sonderpädagogischem Förderbedarf im Gemeinsamen Unterricht. Empirische Sonderpädagogik, 6(1), 59-75.

Krull, J., Wilbert, J., \& Hennemann, T. (2014b). The social and emotional situation of first graders with classroom behavior problems and classroom learning difficulties in inclusive classes. Learning Disabilities: A Contemporary Journal, 12(2), 169-190.

Kulawiak, P.R., \& Wilbert, J. (2015). Methoden zur Analyse der sozialen Integration von Schulkindern mit sonderpädagogischem Förderbedarf im gemeinsamen Unterrich. Empirische Sonderpädagogik, 3, 241-257.

Külker, A., Dorniak, M., Geist, S., Kullmann, H., Lutter, N., \& Lütje-Klose, B. (2017). Schulisches Wohlbefinden als Qualitätsmerkmal inklusiver Schulen - Unterrichtsentwicklung im Rahmen eines Lehrer-Forscher-Projekts an der Laborschule Bielefeld. In A. Textor, S. Grüter, I. Schiermeyer-Reichl \& B. Streese (Hrsg.), Leistung inklusive? Inklusion in der Leistungsgesellschaft (S. 48-59). Bad Heilbrunn: Verlag Julius Klinkhardt.

Kuznetsova, A., Brockhoff, P.B., \& Christensen, R.H.B. (2017). lmertest package: tests in linear mixed effects models. Journal of Statistical Software. https://doi.org/10.18637/jss.v082.i13.

Lackaye, T., Margalit, M., Ziv, O., \& Ziman, T. (2006). Comparisons of self-efficacy, mood, effort, and hope between students with learning disabilities and their non-LD-matched peers. Learning Disabilities Research and Practice, 21(2), 111-121. https://doi.org/10.1111/j.1540-5826.2006.00211.x.

Langford, C.P.H., Bowsher, J., Maloney, J.P., \& Lillis, P. P. (1997). Social support: a conceptual analysis. Journal of Advanced Nursing, 25(1), 95-100. https://doi.org/10.1046/j.1365-2648.1997.1997025095. $\mathrm{X}$.

Lätsch, A. (2017). Do perceived helpfulness and competition in classroom contexts moderate or mediate the association between perceived stress and socio-emotional strengths and difficulties from early to middle adolescence? Learning and Individual Differences, 58, 31-40. https://doi.org/10.1016/j.lindif. 2017.07.006.

Lorger, T., Schmidt, M., \& Vukman, K. B. (2015). The social acceptance of secondary school students with learning disabilities (LD). Bd. 5.

Mand, J. (2007). Social position of special needs pupils in the classroom: a comparison between German special schools for pupils with learning difficulties and integrated primary school classes. European Journal of Special Needs Education, 22(1), 7-14. https://doi.org/10.1080/08856250601082182.

Martschinke, S., Kopp, B., \& Ratz, C. (2012). Gemeinsamer Unterricht von Grundschulkindern und Kindern mit dem Förderschwerpunkt geistige Entwicklung in der ersten Klasse. Erste Ergebnisse einer empirischen Studie zu Effekten auf sozialen Status und soziales Selbstkonzept. Empirische Sonderpädagogik, 4(2), 183-201.

Martínez, R.S. (2006). Social support in inclusive middle schools: perceptions of youth with learning disabilities. Psychology in the Schools, 43(2), 197-209. https://doi.org/10.1002/pits.20142.

McCoy, S., \& Banks, J. (2012). Simply academic? Why children with special educational needs don't like school. European Journal of Special Needs Education, 27(1), 81-97. https://doi.org/10.1080/ 08856257.2011.640487.

Ministerium für Bildung Jugend und Sport (2019). Schule für gemeinsames Lernen. http://www.inklusionbrandenburg.de/startseite.html. Zugegriffen: 13. Aug. 2019.

Ministerium für Bildung Jugend und Sport (2018). Handreichung zur Durchführung des sonderpädagogischen Feststellungsverfahrens. Potsdam: Ministerium für Bildung, Jugend und Sport.

Morin, A. J.S., Maïano, C., Marsh, H. W., Nagengast, B., \& Janosz, M. (2013). School life and adolescents' self-esteem trajectories. Child Development, 84(6), 1967-1988. https://doi.org/10.1111/cdev.12089.

Nakagawa, S., \& Schielzeth, H. (2013). A general and simple method for obtaining R 2 from generalized linear mixed-effects models. (R.B. O’Hara, Hrsg.). Methods in Ecology and Evolution, 4(2), 133-142. https://doi.org/10.1111/j.2041-210x.2012.00261.x. 
Nepi, L.D., Facondini, R., Nucci, F., \& Peru, A. (2013). Evidence from full-inclusion model: the social position and sense of belonging of students with special educational needs and their peers in Italian primary school. European Journal of Special Needs Education, 28(3), 319-332. https://doi.org/10. 1080/08856257.2013.777530.

Nordström, I. (2011). Inclusion and participation in peer relations. Scandinavian Journal of Disability Research, 13(1), 71-87. https://doi.org/10.1080/15017411003711775.

Nota, L., Ferrari, L., \& Soresi, S. (2005). Elementary school children's willingness to help and be friends with disabled peers. International Journal on Disability and Human Development, 4(2), 131-137. https://doi.org/10.1515/IJDHD.2005.4.2.131.

Oswald, H., \& Krappmann, L. (2004). Soziale Ungleichheit in der Schulklasse und Schulerfolg. Zeitschrift für Erziehungswissenschaft, 7(4), 479-496. https://doi.org/10.1007/s11618-004-0055-4.

Patrick, H., Ryan, A. M., \& Kaplan, A. (2007). Early adolescents' perceptions of the classroom social environment, motivational beliefs, and engagement. Journal of Educational Psychology, 99(1), 83-98. https://doi.org/10.1037/0022-0663.99.1.83.

Pepler, D. J., Craig, W. M., \& Roberts, W. L. (1998). Observations of aggressive and nonaggressive children on the school playground. Merrill-Palmer Quarterly, 44(1), 55-76.

Pijl, S. J., \& Frostad, P. (2010). Peer acceptance and self-concept of students with disabilities in regular education. European Journal of Special Needs Education, 25(1), 93-105. https://doi.org/10.1080/ 08856250903450947.

R Development Core Team (2008). R: A language and environment for statistical computing. Wien: R Foundation for Statistical Computing.

Richardson, C. B., Hitti, A., Mulvey, K.L., \& Killen, M. (2014). Social exclusion: the interplay of group goals and individual characteristics. Journal of Youth and Adolescence, 43(8), 1281-1294. https:// doi.org/10.1007/s10964-013-9967-8.

Richardson, C.B., Mulvey, K.L., \& Killen, M. (2012). Extending social domain theory with a processbased account of moral judgments. Human Development, 55(1), 4-25. https://doi.org/10.1159/ 000335362 .

Rost, D.H. (2013). Interpretation und Bewertung pädagogisch-psychologischer Studien. Bad Heilbrunn: Klinkhardt.

Rowe, E.W., Kim, S., Baker, J. A., Kamphaus, R. W., \& Horne, A. M. (2010). Student personal perception of classroom climate: exploratory and confirmatory factor analyses. Educational and Psychological Measurement, 70(5), 858-879. https://doi.org/10.1177/0013164410378085.

von Saldern, M., \& Littig, K.-E. (1987). Landauer Skalen zum Sozialklima. Weinheim: Beltz.

Sauer, S., Ide, S., \& Borchert, J. (2007). Zum Selbstkonzept von Schülerinnen und Schülern an Förderschulen und in integrativer Beschulung: Eine Vergleichsuntersuchung. Heilpädagogische Forschung, 3, 135-142.

Schachner, M. K., Brenick, A., Noack, P., van de Vijver, F. J.R., \& Heizmann, B. (2015). Structural and normative conditions for interethnic friendships in multiethnic classrooms. International Journal of Intercultural Relations, 47, 1-12. https://doi.org/10.1016/j.ijintrel.2015.02.003.

Schachner, M.K., Noack, P., Van de Vijver, F.J.R., \& Eckstein, K. (2016). Cultural Diversity Climate and Psychological Adjustment at School-Equality and Inclusion Versus Cultural Pluralism. Child Development, 87(4), 1175-1191. https://doi.org/10.1111/cdev.12536.

Schubarth, W., \& Speck, K. (2008). Einstellungen, Wohlbefinden, abweichendes Verhalten von Schülerinnen und Schülern. In W. Helsper \& J. Böhme (Hrsg.), Handbuch der Schulforschung (S. 965-984). Wiesbaden: VS. https://doi.org/10.1007/978-3-531-91095-6_40.

Schwab, S. (2014a). Schulische Integration, soziale Partizipation und emotionales Wohlbefinden in der Schule. Ergebnisse einer empirischen Längsschnittstudie. Wien: LIT.

Schwab, S. (2014b). Achievement goals in students with learning disabilities, emotional or behavioral disorders, and low IQ without special educational needs. Journal of Cognitive Education and Psychology, 13(3), 357-374. https://doi.org/10.1891/1945-8959.13.3.357.

Schwab, S. (2019). Friendship stability among students with and without special educational needs. Educational Studies, 45(3), 390-401. https://doi.org/10.1080/03055698.2018.1509774.

Schwab, S., Rossmann, P., Tanzer, N., Hagn, J., Oitzinger, S., Thurner, V., et al. (2015b). Schulisches Wohlbefinden von SchülerInnen mit und ohne sonderpädagogischen Förderbedarf. Zeitschrift für Kinder- und Jugendpsychiatrie und Psychotherapie, 43(4), 265-274. https://doi.org/10.1024/14224917/a000363.

Schwab, S., Gebhardt, M., Krammer, M., \& Gasteiger-Klicpera, B. (2015a). Linking self-rated social inclusion to social behaviour. An empirical study of students with and without special education needs 
in secondary schools. European Journal of Special Needs Education, 30(1), 1-14. https://doi.org/10. 1080/08856257.2014.933550.

Schweer, M. (1997). Bedingungen interpersonalen Vertrauens zum Lehrer: Implizite Vertrauenstheorie, Situationswahrnehmung und Vertrauensaufbau bei Schülern. Psychologie in Erziehung und Unterricht, 44(2), 143-151.

Schwinger, M., Wild, E., Lütje-Klose, B., Grunschel, C., Stranghöner, D., Yotyodying, S., et al. (2015). Wie können motivationale und affektive Merkmale bei Kindern mit sonderpädagogischem Förderbedarf valide erfasst werden? Inklusion von Schülerinnen und Schülern mit sonderpädagogischem Förderbedarf in Schulleistungserhebungen (S. 273-300). Wiesbaden: Springer VS. https://doi.org/ 10.1007/978-3-658-06604-8_10.

Sekretariat der Ständigen Konferenz der Kultusminister der Länder in der Bundesrepublik Deutschland (2018). Sonderpädagogische Förderung in Schulen 2007 bis 2016. Berlin: KMK.

Senatsverwaltung für Bildung Jugend und Familie (2017). Leitfaden zur Feststellung sonderpädagogischen Förderbedarfs an Berliner Schulen. https://www.berlin.de/sen/bildung/schule/foerderung/ sonderpaedagogische-foerderung/fachinfo/leitfaden_foerderbedarf-2017.pdf. Zugegriffen: 8. Aug. 2019.

Senatsverwaltung für Bildung Jugend und Familie (2018). Inklusion. https://www.berlin.de/sen/bildung/ schule/inklusion/. Zugegriffen: 13. Aug. 2019.

Siperstein, G. N., Glick, G. C., \& Parker, R.C. (2009). Social inclusion of children with intellectual disabilities in a recreational setting. Intellectual and Developmental Disabilities, 47(2), 97-107. https:// doi.org/10.1352/1934-9556-47.2.97.

Stelling, S. (2017). Schulisches Wohlbefinden von Kindern mit sonderpädagogischem Förderbedarf Lernen. Eine vergleichende Analyse in inklusiven Klassen und Förderschulklassen des dritten und vierten Jahrgangs. Bielefeld: Universität Bielefeld.

Surachman, A., Wardecker, B., Chow, S.-M., \& Almeida, D. (2018). Life course socioeconomic status, daily stressors, and daily well-being: examining chain of risk models. The Journals of Gerontology: Series B. https://doi.org/10.1093/geronb/gby014.

Torsheim, T., Aaroe, L. E., \& Wold, B. (2003). School-related stress, social support, and distress: prospective analysis of reciprocal and multilevel relationships. Scandinavian Journal of Psychology, 44(2), 153-159. https://doi.org/10.1111/1467-9450.00333.

Vehkakoski, T.M. (2012). 'More homework for me, too'. Meanings of differentiation constructed by elementary-aged students in classroom interaction. European Journal of Special Needs Education, 27(2), 157-170. https://doi.org/10.1080/08856257.2011.645586.

Venetz, M. (2014). Trend: Schulische Integration und Wohlbefinden von Kindern und Jugendlichen mit besonderem Förderbedarf. Vierteljahresschrift für Heilpädagogik und ihre Nachbargebiete, 84(1), 57. https://doi.org/10.2378/vhn2015.art06d.

Venetz, M., Tarnutzer, R., Zurbriggen, C., \& Sempert, W. (2010). Qualität des Erlebens von Lernenden in integrativen und separativen Schulformen: Eine Untersuchung mit der Experience Sampling Method (ESM). Interkantonale Hochschule für Heilpädagogik Zürich. https://www.hfh.ch/fileadmin/ files/documents/Dokumente_FE/D_5_Venetz_2020_Zusammenfassung_D5-1.pdf. Zugegriffen: 25. Juni 2018

Waligora, K. (2002). Der Einfluß sozialer Unterstützung durch Eltern und Peers auf körperliche Beschwerden bei Schülerinnen und Schülern. Praxis der Kinderpsychologie und Kinderpsychiatrie, 2, $178-193$.

Wenz-Gross, M., \& Siperstein, G. N. (1998). Students with learning problems at risk in middle school: stress, social support, and adjustment. Exceptional Children, 65(1), 91-100. https://doi.org/10.1177/ 001440299806500107.

Werning, R., \& Löser, J. M. (2010). Inklusion: aktuelle Diskussionslinien, Widersprüche und Perspektiven. Die deutsche Schule, 102(2), 103-114.

Wild, E., Schwinger, M., Lütje-Klose, B., Yotyodying, S., Gorges, J., Stranghöner, D., et al. (2015). Schülerinnen und Schüler mit dem Förderschwerpunkt Lernen in inklusiven und exklusiven Förderarrangements: Erste Befunde des BiLief-Projektes zu Leistung, sozialer Integration, Motivation und Wohlbefinden. Unterrichtswissenschaft, 43(1), 7-21.

Wocken, H. (1993). Bewältigung von Andersartigkeit: Untersuchungen zur sozialen Distanz in verschiedenen Schulen. In I. P. Gehrmann \& B. Hüwe (Hrsg.), Forschungsprofile der Integration von Behinderten (S. 86-106). Essen: Neue Deutsche Schule.

Wocken, H. (2009). Inklusion \& Integration: Ein Versuch, die Integration vor der Abwertung und die Inklusion vor Träumereien zu bewahren. https://inklusion20.de/material/inklusion/InklusionvsIntegration_ Wocken.pdf. Zugegriffen: 11. Dez. 2019. 